\title{
Loewner chains in the unit disk
}

\author{
Manuel D. Contreras, Santiago Díaz-Madrigal \\ and Pavel Gumenyuk
}

\begin{abstract}
In this paper we introduce a general version of the notion of Loewner chains which comes from the new and unified treatment, given in [5], of the radial and chordal variant of the Loewner differential equation, which is of special interest in geometric function theory as well as for various developments it has given rise to, including the famous Schramm-Loewner evolution. In this very general setting, we establish a deep correspondence between these chains and the evolution families introduced in [5]. Among other things, we show that, up to a Riemann map, such a correspondence is one-to-one. In a similar way as in the classical Loewner theory, we also prove that these chains are solutions of a certain partial differential equation which resembles (and includes as a very particular case) the classical Loewner-Kufarev PDE.
\end{abstract}

\section{Introduction}

\subsection{Classical Loewner theory}

In 1923 Loewner [25] introduced the so-called parametric method in geometric function theory, mainly in the hopes of solving the famous Bieberbach problem about obtaining sharp estimates of Taylor coefficients of normalized holomorphic univalent functions in the unit disk. It is worth recalling that the solution of this problem, given in 1984 by de Branges, relied also on this method. The modern form of the parametric method is mainly due to contributions by Kufarev [18] and Pommerenke [27]. Let us briefly recall the main constructions (see, e.g., [28, Chapter 6]).

2000 Mathematics Subject Classification: Primary 30C80; Secondary 34M15, 30D05. Keywords: Loewner chains, evolution families. 
Let $f_{0}(z)=z+a_{2} z^{2}+\cdots$ be a holomorphic univalent function in the unit disk $\mathbb{D}:=\{z:|z|<1\}$. One can always embed this function into a uniparametric family $\left(f_{t}\right)_{t \geq 0}$ of holomorphic univalent functions in $\mathbb{D}$ satisfying the following two properties: $f_{t}(z)=e^{t} z+a_{2}(t) z^{2}+\cdots$ for any $t \geq 0$ and $f_{s}(\mathbb{D}) \subset f_{t}(\mathbb{D})$ whenever $t \geq s \geq 0$. These type of families are called (classical) Loewner chains. One of the keystones of the parametric method is the fact that every such a family is differentiable in $t$ almost everywhere on $[0,+\infty)$ and independently on $z$. Moreover, they satisfy the following PDE

$$
\frac{\partial f_{t}(z)}{\partial t}=z \frac{\partial f_{t}(z)}{\partial z} p(z, t)
$$

where the driving term $p(z, t)$ is measurable with respect to $t \in[0,+\infty)$ for all $z \in \mathbb{D}$ and holomorphic in $z \in \mathbb{D}$ with $p(0, t)=1$ and $\operatorname{Re} p(z, t)>0$ almost everywhere on $t \in[0,+\infty)$. This equation is called the LoewnerKufarev PDE.

For each $t \geq s \geq 0$, the function $\varphi_{s, t}:=f_{t}^{-1} \circ f_{s}$ is clearly a holomorphic univalent self-mapping of $\mathbb{D}$ and the whole family $\left(\varphi_{s, t}\right)_{t \geq s \geq 0}$ is referred to as the associated evolution family (sometimes transition family or semigroup family) of the Loewner chain. The remarkable fact is that, fixing $z \in \mathbb{D}$ and $s \geq 0$, the functions $w(t)=\varphi_{s, t}(z)$ are integrals of the characteristic equation for (1.1)

$$
\frac{d w}{d t}=-w p(w, t)
$$

with the initial condition $w(s)=z$. This equation is called the LoewnerKufarev $O D E$ and the right member of the equation, the associated vector field. Note that the family $\left(\varphi_{s, t}\right)$ is continuous in $t \in[s,+\infty)$ in the compactopen topology of $\operatorname{Hol}(\mathbb{D}, \mathbb{C})$ for each $s \geq 0$, and satisfies the algebraic conditions

$$
\varphi_{s, s}=i d_{\mathbb{D}}, s \geq 0, \quad \text { and } \quad \varphi_{s, t}=\varphi_{u, t} \circ \varphi_{s, u}, 0 \leq s \leq u \leq t<+\infty .
$$

Another crucial point in the parametric method is that the function $f_{0}$ can be reconstructed by means of the integrals of (1.2). Namely,

$$
\lim _{t \rightarrow+\infty} e^{t} \varphi_{0, t}=f_{0} .
$$

Equation (1.2) can be considered on its own, without any a priori connection to Loewner chains. However, taking any driving term $p(z, t)$ satisfying the above conditions, this equation has a unique solution $w(t)=\varphi_{s, t}(z)$, assuming the initial condition $w(s)=z$. Then, it is possible to define $f_{s}:=\lim _{t \rightarrow+\infty} e^{t} \varphi_{s, t}$ and generate in this way a Loewner chain. Clearly, $\left(\varphi_{s, t}\right)$ is an evolution family associated to this chain $\left(f_{t}\right)$. 
In other words, within the framework of the classical parametric method, there is a one-to-one correspondence between this concept of evolution families, the driving terms (or the vector fields) appearing in Loewner equations and the so-called classical Loewner chains.

\subsection{Chordal Loewner equation}

In his original work [25], Loewner paid special attention to what we call now Loewner chains of slit mappings of the unit disk $\mathbb{D}$. This Loewner chain $\left(f_{t}\right)$ starts with a conformal mapping onto the complex plane minus a Jordan curve going to infinity and, thereafter, the family is obtained by erasing gradually this curve. In this case, the ODE equation (1.2) assumes the following form (see, e.g., [13, chapter III §2] or [12, chapter 3])

$$
\frac{d w}{d t}=-w \frac{\kappa(t)+w}{\kappa(t)-w}
$$

where $\kappa:[0,+\infty) \rightarrow \mathbb{R}$ is a continuous function. The corresponding functions $\varphi_{s, t}=f_{t}^{-1} \circ f_{s}$ map $\mathbb{D}$ onto $\mathbb{D}$ with a slit generated by a Jordan curve starting from the boundary (see [11, Chapter 17]). These self-mappings of the unit disk are normalized at the origin: $\varphi_{s, t}(0)=0, \varphi_{s, t}^{\prime}(0)>0$. However, in many applications, one find quite similar examples but where the natural normalization is at a boundary point of the unit disk. In this case, it is possible to consider a real analogue of (1.4), the chordal Loewner equation (see, e.g., [2, chapter IV $\S 7]$ ), which is traditionally written for the upper half-plane $\mathbb{U}:=\{z: \operatorname{Im} z>0\}$ instead of the unit disk $\mathbb{D}$ because there the associated vector field assumes the simpler form

$$
\frac{d w}{d t}=\frac{2}{\xi(t)-w}, \quad w(0)=z,
$$

where $\xi:[0,+\infty) \rightarrow \mathbb{R}$ is a real-valued driving term. In this chordal context, we could also talk again about driving terms, Loewner chains and evolution families. In contrast to the chordal variant, classical Loewner theory is mentioned in the recent literature as the radial case.

The above chordal variant has been extended to cover a wider variety of new situations. For instance, the relationship between some kind of what we can name chordal Loewner chains and what deserves to be named chordal evolution families has been considered by Goryainov and $\mathrm{Ba}$ in [17] and by Bauer in [3].

A recent burst of interest in Loewner theory is due in part to the so-called Schramm - Loewner evolution (SLE, also known as stochastic Loewner evolution), introduced in 2000 by Schramm [31]. SLE is an evolution model similar to Loewner chains (namely, given by equation (1.4) or (1.5)) but with the 
driving term defined via a Brownian motion. In other words, it is a probabilistic version of the previously known radial and chordal Loewner chains. Both radial and chordal cases of SLE have important applications. In fact, they turn out to be very useful tools for the study of conformally invariant scaling limits of some classical statistical 2-dimensional lattice models, see $[20-24]$.

Some recent developments concerning the relationship between properties of the driving term and the geometry of solutions to (1.4) and (1.5) can be found in $[19,26,30]$.

\subsection{Generalization of classical evolution families}

The Loewner-Kufarev ODE (1.2) defines a holomorphic evolution in the unit disk. That is, for any initial point $z \in \mathbb{D}$ and any starting instance $s \geq 0$, the solution $w=w(t)$ to the initial value problem $w(s)=z$ for equation (1.2) is unique, exists for all $t \geq s$, and the dependence of $w(t)$ on $z$ reveals a holomorphic self-mapping of $\mathbb{D}$. The same is true for the chordal Loewner equation (1.5) and its generalizations when being rewritten for $\mathbb{D}$. Some natural questions arise: are there other examples of ODE with the same property?, what is the most general form of such type of equations?, is it possible to unify these holomorphic evolutions, bearing in mind the many similarities between them?

The answer for the autonomous case (the vector field is of the form $d w / d t=G(w))$ comes from the theory of one-parametric semigroups of holomorphic functions (see the definition in Section 5). They have important applications in the theory of operators acting on spaces of analytic functions (see, e.g., $[32,33]$ ) as well as in the theory of stochastic processes (see, e.g., $[15,16])$. Berkson and Porta [4] found the most general form of such a function $G$, namely

$$
G(z)=(\tau-z)(1-\bar{\tau} z) p(z), \quad z \in \mathbb{D},
$$

where $p$ is a holomorphic function in $\mathbb{D}$ with $\operatorname{Re} p(z) \geq 0$ and $\tau \in \overline{\mathbb{D}}$ (again see Section 5 for more details).

However, in the non-autonomous case and as far as we know, there were no satisfactory answers to the above questions before [5]. Certainly, a large number of examples related to chordal and radial Loewner differential equations has been treated in the literature but, at the same time, one can also find several (similar but different) notions playing the role of Loewner chains, vector fields or, specially, evolution families. For instance, in [14] some classes of holomorphic univalent self-mappings, closed with respect to composition, are considered and evolution families within these classes are defined as twoparametric families $\left(\varphi_{s, t}\right)_{0 \leq s \leq t}$ continuous with respect to $t \in[s,+\infty)$ in the 
open-compact topology of $\operatorname{Hol}(\mathbb{D}, \mathbb{C})$ for each $s \geq 0$ and satisfying the algebraic conditions (1.3). Moreover, in order to describe evolution families by means of differential equations, an additional condition is also imposed: namely, a certain functional applied to $\varphi_{0, t}$ is required to be (locally) absolutely continuous with respect to $t$. It is worth comparing this approach with the very classical case, where one can regard the equality $\varphi_{0, t}^{\prime}(0)=e^{-t}$ as a kind of additional condition ensuring differentiability in $t$.

As we have just partially said, answers to the above questions under very general assumptions follow from results of the recent paper [5] by Bracci and the first two authors of this paper. Taking the whole class of holomorphic self-maps of $\mathbb{D}$, they introduced a general notion of evolution family in the unit disk which includes, as very particular cases, one-parametric semigroups as well as all of those evolution families arising in Loewner theory, both for the radial and chordal variants. Now we cite their definition. Note that the functions $\varphi_{s, t}$ are not assumed a priori to be univalent in $\mathbb{D}$.

Definition 1.1. A family $\left(\varphi_{s, t}\right)_{0 \leq s \leq t<+\infty}$ of holomorphic self-maps of the unit disk is an evolution family of order $d$ with $d \in[1,+\infty]$ (in short, an $L^{d}$-evolution family) if

EF1. $\varphi_{s, s}=i d_{\mathbb{D}}$

EF2. $\varphi_{s, t}=\varphi_{u, t} \circ \varphi_{s, u}$ for all $0 \leq s \leq u \leq t<+\infty$,

EF3. for all $z \in \mathbb{D}$ and for all $T>0$ there exists a non-negative function $k_{z, T} \in L^{d}([0, T], \mathbb{R})$ such that

$$
\left|\varphi_{s, u}(z)-\varphi_{s, t}(z)\right| \leq \int_{u}^{t} k_{z, T}(\xi) d \xi
$$

for all $0 \leq s \leq u \leq t \leq T$.

One of the main results of [5] is that any evolution family $\left(\varphi_{s, t}\right)$ can be obtained via solutions to an ODE of the form $d w / d t=G(w, t)$. Moreover, they characterize all the functions (or, in other words, all the vector fields) $G$ that generate evolution families. Indeed, these vector fields resembles a nonautonomous (the variable $t$ is present) version of the celebrated BerksonPorta representation theorem (see Section 2 for further definitions and full statements of these results). Nevertheless, a one-to-one correspondence between evolution families and certain type of vector fields is established in that paper. There, it is also explained how to recover the semigroup, radial and chordal cases in this new framework. Indeed, the three authors were able to formulate a similar theory of generalized evolution families for arbitrary hyperbolic complex manifolds [6]. 
In [5], the following natural question was implicitly left opened: is there a generalized notion of Loewner chain which can be put in one-to-one correspondence with those generalized evolution families or, equivalently, with those generalized Berkson-Porta vector fields? In the next subsection, we deal with this question presenting our main results about it.

\subsection{Main results}

As we mentioned in Section 1.1, the Loewner-Kufarev equation (1.2) generates a special type of evolution families and there is a one-to-one correspondence between such evolution families and classical Loewner chains.

In this paper we consider the analogous question for arbitrary evolution families in the sense of Definition 1.1. First of all, we give a suitable definition of Loewner chain for our general setting.

Definition 1.2. A family $\left(f_{t}\right)_{0 \leq t<+\infty}$ of holomorphic maps of the unit disk will be called a Loewner chain of order $d$ with $d \in[1,+\infty]$ (in short, an $L^{d}$-Loewner chain) if

LC1. each function $f_{t}: \mathbb{D} \rightarrow \mathbb{C}$ is univalent,

LC2. $f_{s}(\mathbb{D}) \subset f_{t}(\mathbb{D})$ for all $0 \leq s<t<+\infty$,

LC3. for any compact set $K \subset \mathbb{D}$ and all $T>0$ there exists a non-negative function $k_{K, T} \in L^{d}([0, T], \mathbb{R})$ such that

$$
\left|f_{s}(z)-f_{t}(z)\right| \leq \int_{s}^{t} k_{K, T}(\xi) d \xi
$$

for all $z \in K$ and all $0 \leq s \leq t \leq T$.

A Loewner chain $\left(f_{t}\right)$ will be said to be normalized if $f_{0}(0)=0$ and $f_{0}^{\prime}(0)=1$ (notice that we only normalize the function $f_{0}$ ).

Our main results concerning relations between Loewner chains and evolution families are stated in the following three theorems.

Theorem 1.3. For any Loewner chain $\left(f_{t}\right)$ of order $d \in[1,+\infty]$, if we define

$$
\varphi_{s, t}:=f_{t}^{-1} \circ f_{s}, \quad 0 \leq s \leq t,
$$

then $\left(\varphi_{s, t}\right)$ is an evolution family of the same order $d$. Conversely, for any evolution family $\left(\varphi_{s, t}\right)$ of order $d \in[1,+\infty]$, there exists a Loewner chain $\left(f_{t}\right)$ of the same order $d$ such that the following equation holds

$$
f_{t} \circ \varphi_{s, t}=f_{s}, \quad 0 \leq s \leq t .
$$

Definition 1.4. A Loewner chain $\left(f_{t}\right)$ is said to be associated with an evolution family $\left(\varphi_{s, t}\right)$ if it satisfies (1.6). 
Remark 1.5. We will actually prove (see Lemma 3.2) that any Loewner chain $\left(f_{t}\right)$ associated with an evolution family $\left(\varphi_{s, t}\right)$ of order $d \in[1,+\infty]$ must be of the same order $d$.

In general, for a fixed evolution family $\left(\varphi_{s, t}\right)$, the algebraic equation (1.6) does not define a unique Loewner chain. In fact, in some case, plenty of different Loewner chains are associated with the same evolution family. The following theorem gives necessary and sufficient conditions for the uniqueness for a normalized Loewner chain associated with a given evolution family.

Theorem 1.6. Let $\left(\varphi_{s, t}\right)$ be an evolution family. Then there exists a unique normalized Loewner chain $\left(f_{t}\right)$ associated with $\left(\varphi_{s, t}\right)$ such that $\cup_{t \geq 0} f_{t}(\mathbb{D})$ is either an Euclidean disk or the whole complex plane $\mathbb{C}$. Moreover, the following statements are equivalent:

(i) the family $\left(f_{t}\right)$ is the only normalized Loewner chain associated with the evolution family $\left(\varphi_{s, t}\right)$;

(ii) for all $z \in \mathbb{D}$,

$$
\beta(z):=\lim _{t \rightarrow+\infty} \frac{\left|\varphi_{0, t}^{\prime}(z)\right|}{1-\left|\varphi_{0, t}(z)\right|^{2}}=0
$$

(iii) there exist at least one point $z \in \mathbb{D}$ such that $\beta(z)=0$;

(iv) $\bigcup_{t \geq 0} f_{t}(\mathbb{D})=\mathbb{C}$.

The Loewner chain $\left(f_{t}\right)$ in the above theorem will be called the standard Loewner chain associated with the evolution family $\left(\varphi_{s, t}\right)$.

In case of non-uniqueness (when conditions $(i)-(i v)$ in Theorem 1.6 fail to be satisfied), we provide an explicit formula expressing all the associated normalized Loewner chains by means of the standard Loewner chain plus some Riemann map. In some sense, this formula tell us that the evolution procedures described by our Loewner chains are essentially unique up to a choice of the simply connected domain they are located in. Denote by $\mathcal{S}$ the class of all univalent holomorphic functions $h$ in the unit disk $\mathbb{D}$, normalized by $h(0)=h^{\prime}(0)-1=0$.

Theorem 1.7. Suppose that under conditions of Theorem 1.6,

$$
\Omega:=\bigcup_{t \geq 0} f_{t}(\mathbb{D}) \neq \mathbb{C} .
$$

Then $\Omega=\{z:|z|<1 / \beta(0)\}$ and the set $\mathcal{L}\left[\left(\varphi_{s, t}\right)\right]$ of all normalized Loewner chains $\left(g_{t}\right)$ associated with the evolution family $\left(\varphi_{s, t}\right)$, is given by the formula

$$
\mathcal{L}\left[\left(\varphi_{s, t}\right)\right]=\left\{\left(g_{t}\right)_{t \geq 0}: g_{t}(z)=h\left(\beta(0) f_{t}(z)\right) / \beta(0), h \in \mathcal{S}\right\} .
$$


In Section 2 we state some results from [5] along with the necessary definitions. Moreover, we prove new statements concerning evolution families (see Definition 1.1), which we later use to obtain the main results of the paper.

In Section 3 we reformulate and prove the theorems stated above. Namely, Theorem 1.3 follows from Theorems 3.1 and 3.3, while Theorems 1.6 and 1.7 follow from Theorem 3.6 and Proposition 3.4. Besides that, in some cases we establish a necessary and sufficient condition (Theorem 3.8) for a uniparametric family $\left(f_{t}\right)_{t \geq 0}$ of holomorphic (but not a priori univalent) maps defined in $\mathbb{D}$ to be a normalized Loewner chain associated with a given evolution family.

In Section 4 we find an analogue (Theorem 4.1) of the Loewner-Kufarev PDE in this abstract context. We also show that there is a one-to-one correspondence between our concept of generalized Loewner chain and the generalized Berkson-Porta vector fields shown in [5].

In Section 5 we consider the special case of evolution families induced by semigroups of holomorphic functions in $\mathbb{D}$. In particular, we show that the uniqueness of the Kœnigs function is a consequence of Theorems 1.3 and 1.6.

\section{Evolution families and Herglotz vector fields in the unit disk}

Here we collect some known and new statements on evolution families (see Definition 1.1).

Let us first of all note that by [5, Corollary 6.3], given an evolution family $\left(\varphi_{s, t}\right)$, every function $\varphi_{s, t}$ is univalent. The following statement turns out to be also quite useful.

Lemma 2.1. [5, Lemma 3.6] Let $\left(\varphi_{s, t}\right)$ be an evolution family in the unit disk $\mathbb{D}$ of order $d \in[1,+\infty]$. Then for each $0<T<+\infty$ and $0<r<1$, there exists $R=R(r, T)<1$ such that

$$
\left|\varphi_{s, t}(z)\right| \leq R
$$

for all $0 \leq s \leq t \leq T$ and $|z| \leq r$.

Any evolution family $\left(\varphi_{s, t}\right)$ is differentiable almost everywhere with respect to $t$. Besides the proof of this fact, a characterization of all vector fields generating evolution families in the disk is established in [5]. In order to give a strict statement of this result we need the following 
Definition 2.2. Let $d \in[1,+\infty]$. A weak holomorphic vector field of order $d$ in the unit disk $\mathbb{D}$ is a function $G: \mathbb{D} \times[0,+\infty) \rightarrow \mathbb{C}$ with the following properties:

WHVF1. For all $z \in \mathbb{D}$, the function $[0,+\infty) \ni t \mapsto G(z, t)$ is measurable;

WHVF2. For all $t \in[0,+\infty)$, the function $\mathbb{D} \ni z \mapsto G(z, t)$ is holomorphic;

WHVF3. For any compact set $K \subset \mathbb{D}$ and all $T>0$ there exists a nonnegative function $k_{K, T} \in L^{d}([0, T], \mathbb{R})$ such that

$$
|G(z, t)| \leq k_{K, T}(t)
$$

for all $z \in K$ and for almost every $t \in[0, T]$.

Moreover, we say that $G$ is a (generalized) Herglotz vector field (of order $d$ ) if for almost every $t \in[0,+\infty)$ it follows $G(\cdot, t)$ is the infinitesimal generator of a semigroup of holomorphic functions (see Section 5 for further details about semigroups of analytic functions and their infinitesimal generators).

Theorem 2.3. [5, Theorems 6.2, 5.2] For any evolution family $\left(\varphi_{s, t}\right)$ of order $d \in[1,+\infty]$ there exists an (essentially) unique Herglotz vector field $G(z, t)$ of order $d$ such that for all $z \in \mathbb{D}$,

$$
\frac{\partial \varphi_{s, t}(z)}{\partial t}=G\left(\varphi_{s, t}(z), t\right), \quad \text { a.e. } t \in[0,+\infty) .
$$

Conversely, for any Herglotz vector field $G(z, t)$ of order $d \in[1,+\infty]$ there exists a unique evolution family $\left(\varphi_{s, t}\right)$ of order d such that (2.1) is satisfied.

Here by essential uniqueness we mean that two Herglotz vector fields $G_{1}(z, t)$ and $G_{2}(z, t)$ corresponding to the same evolution family must coincide for a.e. $t \geq 0$.

Herglotz vector fields can be further characterized in similar terms of the Berkson - Porta representation of infinitesimal generators.

Definition 2.4. Let $d \in[1,+\infty]$. A Herglotz function of order $d$ is a function $p: \mathbb{D} \times[0,+\infty) \rightarrow \mathbb{C}$ with the following properties:

HF1. For all $z \in \mathbb{D}$, the function $[0,+\infty) \ni t \mapsto p(z, t) \in \mathbb{C}$ belongs to $L_{\text {loc }}^{d}([0,+\infty), \mathbb{C})$;

HF2. For all $t \in[0,+\infty)$, the function $\mathbb{D} \ni z \mapsto p(z, t) \in \mathbb{C}$ is holomorphic;

HF3. For all $z \in \mathbb{D}$ and for all $t \in[0,+\infty)$, we have $\operatorname{Re} p(z, t) \geq 0$. 
Theorem 2.5. [5, Theorem 4.8] Let $G(z, t)$ be a Herglotz vector field of order $d \in[1,+\infty]$ in the unit disk. Then there exist an (essentially) unique measurable function $\tau:[0,+\infty) \rightarrow \overline{\mathbb{D}}$ and a Herglotz function $p(z, t)$ of order $d$ such that for all $z \in \mathbb{D}$

$$
G(z, t)=(z-\tau(t))(\overline{\tau(t)} z-1) p(z, t), \quad \text { a.e. } t \in[0,+\infty) .
$$

Conversely, given a measurable function $\tau:[0,+\infty) \rightarrow \overline{\mathbb{D}}$ and a Herglotz function $p(z, t)$ of order $d \in[1,+\infty]$, equation (2.2) defines a Herglotz vector field of order $d$.

There is thus an (essentially) one-to-one correspondence between evolution families $\left(\varphi_{s, t}\right)$ of order $d \in[1,+\infty]$, Herglotz vector fields $G(z, t)$ of order $d$, and couples $(p, \tau)$ of Herglotz functions $p(z, t)$ of order $d$ and measurable functions $\tau:[0,+\infty) \rightarrow \overline{\mathbb{D}}$. In what follows we say that the couple $(p, \tau)$ is the Berkson-Porta data for $\left(\varphi_{s, t}\right)$.

Now we state and prove some new assertions concerning evolution families, which we use in the proof of the main results.

Denote by $A C^{d}(X, Y), X \subset \mathbb{R}, d \in[1,+\infty]$, the class of all locally absolutely continuous functions $f: X \rightarrow Y$ such that the derivative $f^{\prime}$ belongs to $L_{\text {loc }}^{d}(X)$.

Proposition 2.6. Let $\left(\varphi_{s, t}\right)$ be an evolution family of order $d \in[1,+\infty]$. Then the following statements hold:

1. For any compact set $K \subset \mathbb{D}$ and all $T>0$ there exists a non-negative function $k_{K, T} \in L^{d}([0, T], \mathbb{R})$ such that

$$
\left|\varphi_{s, u}(z)-\varphi_{s, t}(z)\right| \leq \int_{u}^{t} k_{K, T}(\xi) d \xi
$$

for all $0 \leq s \leq u \leq t \leq T$ and all $z \in K$.

2. For every $z \in \mathbb{D}$ the maps $a(t):=\varphi_{0, t}(z)$ and $b(t):=\varphi_{0, t}^{\prime}(z)$ belong to $A C^{d}([0,+\infty), \mathbb{C})$ and $b(t) \neq 0$ for all $t \in[0,+\infty)$.

Proof. By Theorem 2.3, there is a Herglotz vector field of order $d$ such that for all $z \in \mathbb{D}$

$$
\frac{\partial \varphi_{s, t}(z)}{\partial t}=G\left(\varphi_{s, t}(z), t\right), \quad \text { a.e. } t \in[0,+\infty) .
$$

Proof of (1). Let $K$ be a compact set in the unit disk and fix $0<T<+\infty$. By Lemma 2.1, there is $R<1$ such $\left|\varphi_{s, t}(z)\right| \leq R$ for all $z \in K$ and for all 
$0 \leq s \leq t \leq T$. By the very definition of Herglotz vector field there exists a non-negative function $k \in L^{d}([0, T], \mathbb{R})$ such that

$$
|G(z, t)| \leq k(t)
$$

for all $|z| \leq R$ and for almost every $t \in[0, T]$. Therefore, statement (1) is an easy consequence of the following inequalities

$$
\left|\varphi_{s, u}(z)-\varphi_{s, t}(z)\right|=\left|\int_{u}^{t} \frac{\partial \varphi_{s, \xi}(z)}{\partial \xi} d \xi\right|=\left|\int_{u}^{t} G\left(\varphi_{s, \xi}(z), \xi\right) d \xi\right| \leq \int_{u}^{t} k(\xi) d \xi .
$$

Proof of (2). From the very definition of Herglotz vector field, evolution family of order $d$, and inequality (2.3) it follows that the map a belongs to $A C^{d}([0,+\infty), \mathbb{C})$. Moreover, since the functions $\varphi_{s, t}$ are univalent $[5$, Corollary 6.3], we have $b(t) \neq 0$ for all $t$. Fix $T \in(0,+\infty)$ and $z \in \mathbb{D}$. There is $R<1$ such that $\left|\varphi_{0, t}(z)\right|<R$ for all $t \in[0, T]$. Then there is $k_{R, T} \in L^{d}([0, T], \mathbb{R})$ such that

$$
|G(w, t)| \leq k_{R, T}(t)
$$

for all $|w| \leq R$ and for almost every $t \in[0, T]$. Therefore,

$$
\begin{aligned}
\left|b^{\prime}(t)\right| & =\left|\frac{1}{2 \pi} \frac{\partial}{\partial t}\left(\int_{C(0, R)^{+}} \frac{\varphi_{0, t}(w)}{w^{2}} d w\right)\right|=\left|\frac{1}{2 \pi} \int_{C(0, R)^{+}} \frac{\partial}{\partial t}\left(\frac{\varphi_{0, t}(w)}{w^{2}}\right) d w\right| \\
& =\left|\frac{1}{2 \pi}\left(\int_{C(0, R)^{+}} \frac{G\left(\varphi_{0, t}(w), t\right)}{w^{2}} d w\right)\right| \leq \frac{1}{R} k_{R, T}(t)
\end{aligned}
$$

for almost every $t \in[0, T]$, where $C(0, R)^{+}$stands for the positively oriented circle of radius $R$ centered at the point $z=0$. This implies that $b$ belongs to $A C^{d}([0,+\infty), \mathbb{C})$ and therefore completes the proof.

It appears to be useful to consider evolution families that consists of automorphisms of $\mathbb{D}$. The following example is the most general form of such evolution families.

Example 2.7. Take two functions $a, b \in A C^{d}([0,+\infty)$, $\mathbb{C})$ with $|a(t)|<1$ and $|b(t)|=1$ for all $t$ and write

$$
h_{t}(z):=\frac{b(t) z+a(t)}{1+b(t) \overline{a(t)} z} \text { for all } t \geq 0 \text { and all } z \in \mathbb{D} .
$$

Then $\left(h_{t} \circ h_{s}^{-1}\right)$ and $\left(h_{t}^{-1} \circ h_{s}\right)$ are evolution families of order $d$. Indeed, it is clear that both families of functions satisfy EF1 and EF2. Moreover, for any $T<+\infty$ and $z \in \mathbb{D}$ there exists $R<1$ such that

$$
\left|h_{s}^{-1}(z)\right|=\left|\overline{b(s)} \frac{z-a(s)}{1-\overline{a(s)} z}\right| \leq R, \quad 0 \leq s \leq T .
$$


Denote $w=h_{s}^{-1}(z)$. Then we have

$$
\begin{aligned}
& \left|h_{t} \circ h_{s}^{-1}(z)-h_{u} \circ h_{s}^{-1}(z)\right|=\left|h_{t}(w)-h_{u}(w)\right| \\
& =\left|\frac{b(t) w+a(t)}{1+b(t) \overline{a(t)} w}-\frac{b(u) w+a(u)}{1+b(u) \overline{a(u)} w}\right| \\
& \leq \frac{1}{(1-R)^{2}}(|b(t)-b(u)|+|\overline{a(u)} a(t) b(u)-\overline{a(t)} a(u) b(t)|+2|a(t)-a(u)|) \\
& \leq \frac{1}{(1-R)^{2}}(|b(t)-b(u)|+|\overline{a(u)} b(u)-\overline{a(t)} b(t)|+3|a(t)-a(u)|)
\end{aligned}
$$

for all $0 \leq s \leq u \leq t \leq T$. Using the hypothesis on $a$ and $b$, we deduce that the function $\bar{a} b$ belongs to $A C^{d}([0,+\infty), \mathbb{C})$. This fact, the above inequalities, and again the hypothesis on $a$ and $b$ imply that the family $\left(h_{t} \circ h_{s}^{-1}\right)$ satisfies EF3. Similarly, the family $\left(h_{t}^{-1} \circ h_{s}\right)$ satisfies EF3 as well.

The following lemma allows us to transform evolution families by means of time-dependent changes of variable in the unit disk.

Lemma 2.8. Let $\left(\psi_{s, t}\right)$ be an evolution family of order $d \in[1,+\infty]$ and take two functions $a \in A C^{d}([0,+\infty), \mathbb{D})$ and $b \in A C^{d}([0,+\infty), \partial \mathbb{D})$. Write $\varphi_{s, t}=h_{t} \circ \psi_{s, t} \circ h_{s}^{-1}$ and $\tilde{\varphi}_{s, t}=h_{t}^{-1} \circ \psi_{s, t} \circ h_{s}$, where

$$
h_{t}(z):=\frac{b(t) z+a(t)}{1+b(t) \overline{a(t)} z} \text { for all } t \geq 0 \text { and all } z \in \mathbb{D} .
$$

Then $\left(\varphi_{s, t}\right)$ and $\left(\tilde{\varphi}_{s, t}\right)$ are evolution families of order $d$.

Proof. We present the proof for the family $\left(\varphi_{s, t}\right)$ and leave to the reader the one for the family $\left(\tilde{\varphi}_{s, t}\right)$ which is quite similar.

It is clear that the functions $\left(\varphi_{s, t}\right)$ satisfy properties EF1 and EF2. So we just have to prove that this family of functions satisfy EF3.

Notice that, by Example 2.7, $\left(h_{t} \circ h_{s}^{-1}\right)$ is an evolution family. Fix $z \in \mathbb{D}$ and $T \in(0, \infty)$. By Lemma 2.1 and the continuity of the functions $a$ and $b$, there exists a number $R<1$ such that

$$
\left|\psi_{s, t} \circ h_{s}^{-1}(z)\right| \leq R \text { and }\left|\varphi_{s, t}(z)\right|=\left|h_{t} \circ \psi_{s, t} \circ h_{s}^{-1}(z)\right| \leq R
$$

for all $0 \leq s \leq t \leq T$. Therefore, by Proposition 2.6 applied to the evolution families $\left(h_{t} \circ h_{s}^{-1}\right)$ and $\left(\psi_{s, t}\right)$, there are two functions $k_{1}, k_{2} \in L^{d}([0, T], \mathbb{R})$ such that

$$
\begin{aligned}
& \left|\psi_{s, u}(w)-\psi_{s, t}(w)\right| \leq \int_{u}^{t} k_{1}(\xi) d \xi \quad \text { and } \\
& \left|h_{u} \circ h_{s}^{-1}(w)-h_{t} \circ h_{s}^{-1}(w)\right| \leq \int_{u}^{t} k_{2}(\xi) d \xi
\end{aligned}
$$


for all $0 \leq s \leq u \leq t \leq T$ and whenever $|w| \leq R$. Moreover, there is a positive number $M$ such that

$$
\left|h_{t}\left(w_{1}\right)-h_{t}\left(w_{2}\right)\right| \leq M\left|w_{1}-w_{2}\right|
$$

whenever $t \in[0, T]$ and $\left|w_{1}\right|,\left|w_{2}\right| \leq R$. Now, let us fix $0 \leq s \leq u \leq t \leq T$ and write $z_{1}=\psi_{s, u}\left(h_{s}^{-1}(z)\right)$ and $z_{2}=h_{u}\left(z_{1}\right)$. Note that $\left|z_{1}\right|,\left|z_{2}\right| \leq R$. The following chain of inequalities (where we use (2.4) and (2.5)) allows us to complete the proof

$$
\begin{aligned}
\left|\varphi_{s, t}(z)-\varphi_{s, u}(z)\right| & =\left|\varphi_{u, t}\left(\varphi_{s, u}(z)\right)-\varphi_{s, u}(z)\right|=\left|h_{t} \circ \psi_{u, t}\left(z_{1}\right)-h_{u}\left(z_{1}\right)\right| \\
& \leq\left|h_{t} \circ \psi_{u, t}\left(z_{1}\right)-h_{t}\left(z_{1}\right)\right|+\left|h_{t}\left(z_{1}\right)-h_{u}\left(z_{1}\right)\right| \\
& \leq M\left|\psi_{u, t}\left(z_{1}\right)-z_{1}\right|+\left|h_{t}\left(z_{1}\right)-h_{u}\left(z_{1}\right)\right| \\
& =M\left|\psi_{u, t}\left(z_{1}\right)-\psi_{u, u}\left(z_{1}\right)\right|+\left|h_{t} \circ h_{u}^{-1}\left(z_{2}\right)-h_{u} \circ h_{u}^{-1}\left(z_{2}\right)\right| \\
& \leq \int_{u}^{t}\left(M k_{1}(\xi)+k_{2}(\xi)\right) d \xi .
\end{aligned}
$$

Now we use Lemma 2.8 in order to establish a kind of decomposition for a given evolution family.

Proposition 2.9. Let $\left(\varphi_{s, t}\right)$ be an evolution family of order $d \in[1,+\infty]$. Then there exist unique $a \in A C^{d}([0,+\infty), \mathbb{D}), b \in A C^{d}([0,+\infty), \partial \mathbb{D})$, and $\psi_{s, t}: \mathbb{D} \rightarrow \mathbb{D}, 0 \leq s \leq t<+\infty$, such that the following assertions hold

1. $a(0)=0, b(0)=1$,

2. $\left(\psi_{s, t}\right)$ is an evolution family of order $d$ such that $\psi_{s, t}(0)=0$ and $\psi_{s, t}^{\prime}(0)>0$ for all $0 \leq s \leq t$,

3. $\varphi_{s, t}=h_{t} \circ \psi_{s, t} \circ h_{s}^{-1}$ for all $0 \leq s \leq t<+\infty$, where

$$
h_{t}(z):=\frac{b(t) z+a(t)}{1+b(t) \overline{a(t)} z}, \quad t \geq 0, \quad z \in \mathbb{D} .
$$

Proof. Write $a(t)=\varphi_{0, t}(0)$ and $b(t)=\frac{\varphi_{0, t}^{\prime}(0)}{\left|\varphi_{0, t}^{\prime}(0)\right|}$. By Proposition 2.6, $a \in$ $A C^{d}([0,+\infty), \mathbb{D})$ and $b \in A C^{d}([0,+\infty), \partial \mathbb{D})$. Now define $h_{t}$ as in the statement of the proposition and take $\psi_{s, t}=h_{t}^{-1} \circ \varphi_{s, t} \circ h_{s}$. Notice that $h_{0}$ is the identity, $h_{t}(0)=a(t)$ and $h_{t}^{\prime}(0)=b(t)\left(1-|a(t)|^{2}\right)$. By Lemma 2.8, the family $\left(\psi_{s, t}\right)$ is an evolution family of order $d$. Moreover, from the very definition of $a$ it follows that $\psi_{0, t}(0)=0$ for all $t$. Using EF2, we deduce that $\psi_{s, t}(0)=0$ for all $s \leq t$. In a similar way, we show that $\psi_{0, t}^{\prime}(0)=\frac{\left|\varphi_{0, t}^{\prime}(0)\right|}{1-|a(t)|^{2}}>0$ for all $t$ and then $\psi_{s, t}^{\prime}(0)>0$ for all $0 \leq s \leq t$. 
The uniqueness is clear because from the equality $\varphi_{s, t}=h_{t} \circ \psi_{s, t} \circ h_{s}^{-1}$ we deduce that $a(t)=h_{t}(0)=h_{t}\left(\psi_{0, t}(0)\right)=\varphi_{0, t}(0), b(t)=\frac{\varphi_{0, t}^{\prime}(0)}{\left|\varphi_{0, t}^{\prime}(0)\right|}$ (which defines the functions $h_{t}$ uniquely) and $\psi_{s, t}=h_{t}^{-1} \circ \varphi_{s, t} \circ h_{s}$. The proof is now complete.

The following result gives the converse of Proposition 2.6(2).

Proposition 2.10. Let $\left(\varphi_{s, t}\right)$ be a family of holomorphic self-maps of $\mathbb{D}$. Suppose that conditions EF1 and EF2 are fulfilled. Then condition EF3 is equivalent to the following condition:

EF4. The maps $a(t):=\varphi_{0, t}(0)$ and $b(t):=\varphi_{0, t}^{\prime}(0)$ belong to $A C^{d}([0,+\infty), \mathbb{C})$ and $b(t) \neq 0$ for all $t \in[0,+\infty)$.

Proof. By Proposition 2.6 any evolution family satisfies EF4.

Let $\left(\varphi_{s, t}\right)$ be a family of holomorphic self-maps of the unit disk satisfying EF1, EF2, and EF4. Write

$$
h_{t}(z):=\frac{b_{0}(t) z+a(t)}{1+b_{0}(t) \overline{a(t)} z} \quad \text { for all } t \geq 0 \text { and all } z \in \mathbb{D}
$$

where $b_{0}(t)=b(t) /|b(t)|$. Define $\psi_{s, t}=h_{t}^{-1} \circ \varphi_{s, t} \circ h_{s}$ for all $0 \leq s \leq t<$ $+\infty$. It is clear that the family $\left(\psi_{s, t}\right)$ satisfies EF1, EF2, $\psi_{s, t}(0)=0$, and $\psi_{0, t}^{\prime}(0)=|b(t)| /\left(1-|a(t)|^{2}\right)$ for all $0 \leq s \leq t$. Using [5, Theorem 7.3] with $\tau=z_{0}=0$ in that statement, we deduce that $\left(\psi_{s, t}\right)$ is an evolution family of order $d$. Finally, we just have to apply Lemma 2.8 to deduce that $\left(\varphi_{s, t}\right)$ is also an evolution family of order $d$.

\section{Loewner chains and evolution families}

In this section we reformulate and prove our main results connecting evolution families with Loewner chains in a way similar to the one given in classical Loewner theory.

First of all we prove that any Loewner chain of order $d \in[0,+\infty]$ generates an evolution family of the same order.

Theorem 3.1. Let $\left(f_{t}\right)$ be a Loewner chain of order $d \in[1,+\infty]$. Set

$$
\varphi_{s, t}(z):=f_{t}^{-1}\left(f_{s}(z)\right), z \in \mathbb{D}, 0 \leq s \leq t .
$$

Then $\left(\varphi_{s, t}\right)$ is a well-defined evolution family of order $d$ in the unit disk and (trivially) satisfies the equality

$$
f_{t}\left(\varphi_{s, t}(z)\right)=f_{s}(z), z \in \mathbb{D}, 0 \leq s \leq t .
$$


Proof . The proof of this theorem is quite long so we have divided it into several steps of independent interest on their own. In what follows, $\Omega_{t}:=$ $f_{t}(\mathbb{D}), t \geq 0$. We also comment that ins $\Gamma$ will denote the interior of a Jordan curve $\Gamma$ and $N(g, \Gamma)$ stands for the number of zeros (counting multiplicity), inside a rectifiable Jordan curve $\Gamma$ contained in $\mathbb{D}$, of a holomorphic map $g$ defined in the whole unit disk. Finally, by ind $(\Gamma, \xi)$ we denote the index of a closed rectifiable curve $\Gamma$ with respect to a point $\xi$, and $D(\xi, r):=\{z \in$ $\mathbb{C}:|z-\xi|<r\}$.

[Step 1] For every $t \geq 0$ and every $\omega \in \Omega_{t}$, there exist $\varepsilon>0, \delta>0$ and a rectifiable Jordan curve $\gamma$ with $\gamma \cup$ ins $\gamma \subset \mathbb{D}$ such that the following "locally uniform formula for the inverses" holds:

$$
f_{u}^{-1}(w)=\frac{1}{2 \pi i} \int_{\gamma} \frac{\xi f_{u}^{\prime}(\xi)}{f_{u}(\xi)-w} d \xi
$$

whenever $u \in[t-\delta, t+\delta] \cap[0,+\infty)$ and $w \in D(\omega, \varepsilon)$.

Fix $t \geq 0$ and $\omega \in \Omega_{t}$. Denote $z_{0}:=f_{t}^{-1}(\omega) \in \mathbb{D}$ and choose any $r \in$ $\left(\left|z_{0}\right|, 1\right)$ and $R \in(r, 1)$. Consider the complex domain $D_{t}:=f_{t}(D(0, r)) \subset \Omega_{t}$ and define $\gamma$ as the positively oriented circle of radius $R$ centered at the origin. Since $f_{t}$ is univalent, it follows from the Argument Principle that for each $w \in D_{t}$,

$$
\frac{1}{2 \pi i} \int_{\gamma} \frac{f_{t}^{\prime}(\xi)}{f_{t}(\xi)-w} d \xi=N\left(f_{t}-w, \gamma\right)=1
$$

Note that

$$
\inf \left\{\left|w-f_{t}(z)\right|: w \in \overline{D_{t}},|z|=R\right\}>0,
$$

because $r<R$ and $f_{t}$ is continuous and univalent in $\mathbb{D}$. Moreover, by property LC3, we know that $f_{s} \rightarrow f_{t}$ uniformly on $\overline{D(0, R)}$ as $s \rightarrow t$. This implies the existence of a number $\delta_{0}>0$ such that

$$
\inf \left\{\left|w-f_{u}(z)\right|: w \in \overline{D_{t}},|z|=R\right\}>0,
$$

for all non-negative $u \in\left[t-\delta_{0}, t+\delta_{0}\right]$. In particular, this allows to consider, for every $w \in D_{t}$ and every non-negative $u \in\left[t-\delta_{0}, t+\delta_{0}\right]$, the Argument Principle formula

$$
\frac{1}{2 \pi i} \int_{\gamma} \frac{f_{u}^{\prime}(\xi)}{f_{u}(\xi)-w} d \xi=N\left(f_{u}-w, \gamma\right)
$$

Again, using property LC3 and the Weierstrass Theorem, we conclude that

$$
\lim _{u \rightarrow t} \sup \left\{\left|N\left(f_{u}-w, \gamma\right)-1\right|: w \in D_{t}\right\}=0 .
$$


But $N\left(f_{u}-w, \gamma\right)$ can take only integer values, so there exists $\delta_{1} \in\left(0, \delta_{0}\right)$ such that

$$
\sup \left\{\left|N\left(f_{u}-w, \gamma\right)-1\right|: w \in D_{t}\right\}=0,
$$

whenever $u \in\left[t-\delta_{1}, t+\delta_{1}\right] \cap[0,+\infty)$. In other words, we have showed that

$$
N\left(f_{u}-w, \gamma\right)=1 \text {, when } u \in\left[t-\delta_{1}, t+\delta_{1}\right] \cap[0,+\infty) \text { and } w \in D_{t} .
$$

At this point, we fix $u \in\left[t-\delta_{1}, t+\delta_{1}\right]$ and $w \in D_{t}$. Our idea is to apply now the generalized Argument Principle for the couple $\left(i d, f_{u}-w\right)$ and the rectifiable closed curve $\gamma$ (see, e.g., [10, p. 124, chapter V, Theorem 3.6]). Namely, recalling that $f_{u}-w$ is analytic in the unit disk with a unique zero (denoted by $f_{u}^{-1}(w)$ ) which is contained in ins $\gamma$, we deduce that

$$
\frac{1}{2 \pi i} \int_{\gamma} i d(\xi) \frac{f_{u}^{\prime}(\xi)}{f_{u}(\xi)-w} d \xi=i d\left(f_{u}^{-1}(w)\right) N\left(f_{u}-w, \gamma\right)=f_{u}^{-1}(w) .
$$

In order to finish the proof of Step 1 it is enough to define $\varepsilon$ as the distance between $\omega$ and the boundary of $D_{t}$, which is positive since $D_{t}$ is open and $\omega \in D_{t}$ by construction.

[Step 2] For any $r \in(0,1)$ and any $T>0$, we have that

$$
\sup \left\{\left|\left(f_{t}^{-1} \circ f_{s}\right)(z)\right|: 0 \leq s \leq t \leq T,|z| \leq r\right\}<1 .
$$

Fix $r \in(0,1)$ and $T>0$ and suppose that the above supremum is 1 . Then, there exist sequences $\left(s_{n}\right),\left(t_{n}\right)$ and $\left(z_{n}\right)$ such that:

(a) for all $n \in \mathbb{N}, 0 \leq s_{n} \leq t_{n} \leq T,\left|z_{n}\right| \leq r$,

(b) the following limits exist $s:=\lim _{n} s_{n}, t:=\lim _{n} t_{n}, z_{0}:=\lim _{n} z_{n}, \beta:=$ $\lim _{n}\left(f_{t_{n}}^{-1} \circ f_{s_{n}}\right)\left(z_{n}\right)$, and

(c) $0 \leq s \leq t \leq T,\left|z_{0}\right| \leq r, \beta \in \partial \mathbb{D}$.

We note that $f_{s}\left(z_{0}\right) \in \Omega_{s} \subset \Omega_{t}$ and $\lim _{n} f_{s_{n}}\left(z_{n}\right)=f_{s}\left(z_{0}\right)$. Therefore, by [Step 1], there exist $\varepsilon>0, \delta>0$ and a Jordan curve $\gamma$ with $\gamma \cup$ ins $\gamma \subset \mathbb{D}$ such that

$$
f_{u}^{-1}(w)=\frac{1}{2 \pi i} \int_{\gamma} \frac{\xi f_{u}^{\prime}(\xi)}{f_{u}(\xi)-w} d \xi
$$

whenever $u \in[t-\delta, t+\delta] \cap[0,+\infty)$ and $w \in D\left(f_{s}\left(z_{0}\right), \varepsilon\right)$. In particular, for $n$ large enough, we have that

$$
f_{t_{n}}^{-1}\left(f_{s_{n}}\left(z_{n}\right)\right)=\frac{1}{2 \pi i} \int_{\gamma} \frac{\xi f_{t_{n}}^{\prime}(\xi)}{f_{t_{n}}(\xi)-f_{s_{n}}\left(z_{n}\right)} d \xi .
$$


Clearly, by property LC3 and the above formula

$$
\begin{aligned}
f_{t_{n}}^{-1}\left(f_{s_{n}}\left(z_{n}\right)\right)= & \frac{1}{2 \pi i} \int_{\gamma} \frac{\xi f_{t_{n}}^{\prime}(\xi)}{f_{t_{n}}(\xi)-f_{s_{n}}\left(z_{n}\right)} d \xi \\
& \longrightarrow \frac{1}{2 \pi i} \int_{\gamma} \frac{\xi f_{t}^{\prime}(\xi)}{f_{t}(\xi)-f_{s}\left(z_{0}\right)} d \xi=f_{t}^{-1}\left(f_{s}\left(z_{0}\right)\right)
\end{aligned}
$$

as $n \rightarrow+\infty$. Since $f_{t}^{-1}\left(f_{s}\left(z_{0}\right)\right) \in \mathbb{D}$, we obtain a contradiction, which finishes the proof of Step 2.

[Step 3] Let $\gamma:[a, b] \rightarrow \mathbb{C}$ be a rectifiable curve in $\mathbb{D}$ and $T>0$. Then, for all $t \in[0, T]$, the curve

$$
\gamma_{t}:[a, b] \rightarrow \mathbb{C}, \xi \mapsto f_{t}(\gamma(\xi)) \in \Omega_{t}
$$

is a well-defined rectifiable curve in $\Omega_{t}$. Moreover,

$$
\sup \left\{\operatorname{len}\left(\gamma_{t}\right): t \in[0, T]\right\}<+\infty,
$$

where, as usual, len $\left(\gamma_{t}\right)$ denotes the length of $\gamma_{t}$.

The fact that $\gamma_{t}$ is a well-defined rectifiable curve is widely known. So, suppose that the above supremum is $+\infty$. In this case, there exists a sequence $\left(t_{n}\right)$ in the interval $[0, T]$ such that $\lim _{n} t_{n}=t \in[0, T]$ and $\lim _{n} \operatorname{len}\left(\gamma_{t_{n}}\right)=$ $+\infty$. However, the well-known estimate

$$
\operatorname{len}\left(\gamma_{t_{n}}\right) \leq \operatorname{len}(\gamma) \max \left\{\left|f_{t_{n}}^{\prime}(\xi)\right|: \xi \in \gamma\right\}
$$

shows (recall that $\gamma$ is a compact set) that there exists a subsequence $\left(z_{n_{k}}\right)$ in the curve $\gamma$ converging to some $z_{0} \in \gamma$ such that $\lim _{k} f_{t_{n_{k}}}^{\prime}\left(z_{n_{k}}\right)=\infty$. However, by property LC3 and Weierstrass' Theorem, we deduce $\lim _{k} f_{t_{n_{k}}}^{\prime}\left(z_{n_{k}}\right)=$ $f_{t}^{\prime}\left(z_{0}\right)$, obtaining in this way a contradiction.

[Step 4] In this step we will finally prove the theorem.

By properties LC1 and LC2, we see that the functions

$$
\varphi_{s, t}(z):=f_{t}^{-1}\left(f_{s}(z)\right), z \in \mathbb{D}, 0 \leq s \leq t
$$

are well-defined and, indeed, $\varphi_{s, t} \in \operatorname{Hol}(\mathbb{D}, \mathbb{D})$, for any $0 \leq s \leq t$. Hence, $\left(\varphi_{s, t}\right)$ will be an evolution family of order $d$ if we are able to prove properties EF1, EF2, and EF3. The first two properties follow easily from the way we have defined the family $\left(\varphi_{s, t}\right)$. The third property is more difficult to prove. 
We fix $z \in \mathbb{D}$ and $T>0$. By [Step 2], there exists $R_{1}:=R_{1}(z, T) \in(0,1)$ such that

$$
\sup \left\{\left|\varphi_{a, b}(z)\right|: 0 \leq a \leq b \leq T\right\} \leq R_{1} .
$$

Applying again [Step 2], we obtain another $R_{2}:=R_{2}(z, T) \in(0,1)$ such that $R_{2}>R_{1}$ and

$$
\sup \left\{\left|\varphi_{a, b}(\xi)\right|: 0 \leq a \leq b \leq T,|\xi| \leq R_{1}\right\}<R_{2}
$$

Additionally, we denote by $\gamma$ the positively oriented circle of radius $R_{2}$ centered at the origin. As in [Step 3], we also consider the rectifiable curves $\gamma_{t}:=f_{t} \circ \gamma$, which are Jordan curves due to the univalence of $f_{t}$.

Now, assume that $0 \leq s \leq u \leq t \leq T$. Then, using property EF2, we obtain

$$
\left|\varphi_{s, u}(z)-\varphi_{s, t}(z)\right|=\left|\varphi_{s, u}(z)-\varphi_{u, t}\left(\varphi_{s, u}(z)\right)\right| \leq \sup \left\{\left|\varphi_{u, t}(\xi)-\xi\right|:|\xi| \leq R_{1}\right\}
$$

But, for any $|\xi| \leq R_{1}$, we have that $\left|f_{t}^{-1}\left(f_{u}(\xi)\right)\right|<R_{2}$, so $f_{u}(\xi) \in f_{t}($ ins $\gamma)$. Applying [28, Lemma 1.1], we see that $f_{u}(\xi) \in \operatorname{ins} \gamma_{t}$. The same argument shows that $f_{t}(\xi) \in$ ins $\gamma_{t}$. Therefore, using the Cauchy Integral Formula, for all $|\xi| \leq R_{1}$ we get

$$
\begin{aligned}
\mid f_{t}^{-1}\left(f_{u}(\xi)\right) & -\xi|=| f_{t}^{-1}\left(f_{u}(\xi)\right)-f_{t}^{-1}\left(f_{t}(\xi)\right) \mid \\
& =\left|\frac{\operatorname{ind}\left(\gamma_{t}, f_{u}(\xi)\right)}{2 \pi i} \int_{\gamma_{t}} \frac{f_{t}^{-1}(\eta) d \eta}{\eta-f_{u}(\xi)}-\frac{\operatorname{ind}\left(\gamma_{t}, f_{t}(\xi)\right)}{2 \pi i} \int_{\gamma_{t}} \frac{f_{t}^{-1}(\eta) d \eta}{\eta-f_{t}(\xi)}\right| \\
& \leq \frac{1}{2 \pi}\left|f_{u}(\xi)-f_{t}(\xi)\right|\left|\int_{\gamma_{t}} \frac{f_{t}^{-1}(\eta)}{\left(\eta-f_{u}(\xi)\right)\left(\eta-f_{t}(\xi)\right)} d \eta\right| .
\end{aligned}
$$

We claim that

$$
l=l(z, T):=\inf \left\{\left|f_{t}(a)-f_{u}(b)\right|: 0 \leq u \leq t \leq T,|a|=R_{2},|b| \leq R_{1}\right\}>0 .
$$

Therefore, recalling that $f_{t}^{-1}\left(\Omega_{t}\right) \subset \mathbb{D}$ and using the above estimation, we have

$$
\left|f_{t}^{-1}\left(f_{u}(\xi)\right)-\xi\right| \leq \frac{1}{2 \pi}\left|f_{u}(\xi)-f_{t}(\xi)\right| \frac{1}{l^{2}} \operatorname{len}\left(\gamma_{t}\right) .
$$

Now, by [Step 3], there exists $C=C(z, T)>0$ such that

$$
\sup \left\{\operatorname{len}\left(\gamma_{t}\right): t \in[0, T]\right\} \leq C,
$$

SO

$$
\left|\varphi_{s, u}(z)-\varphi_{s, t}(z)\right| \leq \frac{C}{2 \pi l^{2}} \sup \left\{\left|f_{u}(\xi)-f_{t}(\xi)\right|:|\xi| \leq R_{1}\right\}
$$


Finally, by property LC3 with $K:=\overline{D\left(0, R_{1}\right)}$, there exists a non-negative function $k_{z, T} \in L^{d}([0, T] ; \mathbb{R})$ such that

$$
\left|\varphi_{s, u}(z)-\varphi_{s, t}(z)\right| \leq \frac{C}{2 \pi l^{2}} \int_{u}^{t} k(\eta) d \eta .
$$

Now it remains to prove that $l>0$. Suppose on the contrary that $l=0$. Then, there exist sequences $\left(a_{n}\right),\left(b_{n}\right),\left(u_{n}\right)$ and $\left(t_{n}\right)$ such that:

(a) for all $n \in \mathbb{N}, 0 \leq u_{n} \leq t_{n} \leq T,\left|a_{n}\right|=R_{2},\left|b_{n}\right| \leq R_{1}$,

(b) there exist the following limits $u:=\lim _{n} u_{n}, t:=\lim _{n} t_{n}, a:=\lim _{n} a_{n}$, $b:=\lim _{n} b_{n}$,

(c) $0 \leq u \leq t \leq T,|a|=R_{2},|b| \leq R_{1}$, and

(d) $f_{t_{n}}\left(a_{n}\right)-f_{u_{n}}\left(b_{n}\right) \rightarrow 0$ as $n \rightarrow+\infty$.

By property LC3, we know that $\left(f_{u_{n}}\right)$ and $\left(f_{t_{n}}\right)$ tends to $f_{u}$ and $f_{t}$, respectively, in the compact-open topology of $\operatorname{Hol}(\mathbb{D}, \mathbb{C})$. Therefore, by $(b)$ and $(d)$, we conclude that $f_{u}(b)=f_{t}(a)$. However, using $(c)$ from the definition of the Jordan curves $\gamma$ and $\gamma_{t}$ it is clear that $a \in \gamma$ and $f_{t}(a) \in f_{t} \circ \gamma=\gamma_{t}$. At the same time, $|b| \leq R_{1}$. So by the choice of $R_{2}$ we find that $\left|f_{t}^{-1}\left(f_{u}(b)\right)\right|<R_{2}$. Thus, $f_{u}(b) \in f_{t}($ ins $\gamma)=\operatorname{ins} \gamma_{t}$ by [28, Lemma 1.1]. Obviously $\gamma_{t} \cap \operatorname{ins} \gamma_{t}=\emptyset$, so we have a contradiction, which finishes the proof.

The following lemma shows that if an evolution family has order $d \in[1,+\infty]$, then any Loewner chain associated with it is also of order $d$. From another point of view, the next lemma shows that the algebraic equation (1.6) implies indirectly conditions LC2 and LC3.

Lemma 3.2. Let $\left(\varphi_{s, t}\right)$ be an evolution family of order $d \in[1,+\infty]$. Assume that for all $t \geq 0$ the function $f_{t}: \mathbb{D} \rightarrow \mathbb{C}$ is univalent and

$$
f_{t} \circ \varphi_{s, t}=f_{s}, \quad 0 \leq s \leq t<+\infty .
$$

Then the family $\left(f_{t}\right)$ is a Loewner chain of order $d$.

Proof. Let $K$ be a compact subset of $\mathbb{D}$ and $T>0$. By Lemma 2.1, there exists $R_{1} \in(0,1)$ such that $\left|\varphi_{s, t}(z)\right| \leq R_{1}$ for all $z \in K$ whenever $0 \leq s \leq$ $t \leq T$. Write $R_{2}=\left(1+R_{1}\right) / 2$. Again by Lemma 2.1 , there exists $R_{3} \in(0,1)$ such that $\left|\varphi_{s, t}(z)\right| \leq R_{3}$ for all $|z|=R_{2}$ and all $0 \leq s \leq t \leq T$. Since the function $f_{T}$ is continuous, there is a positive constant $M$ such that

$$
\left|f_{t}(\xi)\right|=\left|f_{T}\left(\varphi_{t, T}(\xi)\right)\right| \leq M
$$

for all $t \leq T$ and any complex number $\xi$ with $|\xi|=R_{2}$. 
Fix $z \in K$ and $0 \leq s \leq t \leq T$. We have

$$
\begin{aligned}
f_{s}(z)-f_{t}(z) & =f_{t}\left(\varphi_{s, t}(z)\right)-f_{t}(z) \\
& =\frac{1}{2 \pi i} \int_{C\left(0, R_{2}\right)^{+}}\left(\frac{f_{t}(\xi)}{\xi-\varphi_{s, t}(z)}-\frac{f_{t}(\xi)}{\xi-z}\right) d \xi \\
& =\frac{1}{2 \pi i} \int_{C\left(0, R_{2}\right)^{+}}\left(\frac{f_{t}(\xi)\left(\varphi_{s, t}(z)-z\right)}{\left(\xi-\varphi_{s, t}(z)\right)(\xi-z)}\right) d \xi \\
& =\frac{\varphi_{s, t}(z)-z}{2 \pi i} \int_{C\left(0, R_{2}\right)^{+}}\left(\frac{f_{t}(\xi)}{\left(\xi-\varphi_{s, t}(z)\right)(\xi-z)}\right) d \xi
\end{aligned}
$$

Therefore,

$$
\begin{aligned}
\left|f_{s}(z)-f_{t}(z)\right| & =\left|\frac{\varphi_{s, t}(z)-z}{2 \pi i} \int_{C\left(0, R_{2}\right)^{+}}\left(\frac{f_{t}(\xi)}{\left(\xi-\varphi_{s, t}(z)\right)(\xi-z)}\right) d \xi\right| \\
& \leq R_{2} \frac{M}{\left(R_{2}-R_{1}\right)^{2}}\left|\varphi_{s, t}(z)-z\right|
\end{aligned}
$$

for all $z \in K$ and $0 \leq s \leq t \leq T$. Now the conclusion of the lemma easily follows from the last inequality.

Now we prove the existence of a Loewner chain associated with a given evolution family.

Theorem 3.3. Let $\left(\varphi_{s, t}\right)$ be an evolution family of order $d \in[1,+\infty]$. Then there exists a normalized Loewner chain $\left(f_{t}\right)$ of order $d$ associated with the evolution family $\left(\varphi_{s, t}\right)$ such that the set $\Omega:=\cup_{t \geq 0} f_{t}(\mathbb{D})$ coincides with the disk $\{z:|z|<1 / \beta\}$ if $\beta>0$ and with the whole complex plane $\mathbb{C}$ if $\beta=0$, where

$$
\beta=\lim _{t \rightarrow+\infty} \frac{\left|\varphi_{0, t}^{\prime}(0)\right|}{1-\left|\varphi_{0, t}(0)\right|^{2}} .
$$

Proof. By Proposition 2.9 we have $\varphi_{s, t}=h_{t} \circ \psi_{s, t} \circ h_{s}^{-1}$, where $\left(\psi_{s, t}\right)$ is an evolution family such that $\psi_{s, t}(0)=0$ and $\psi_{s, t}^{\prime}(0)>0$ for all $t \geq s \geq 0$, and $h_{t}$ is a conformal automorphism of $\mathbb{D}$ for each $t \geq 0$, with $h_{0}$ being the identity map.

Now we build the Loewner chain for the evolution family $\left(\psi_{s, t}\right)$ and then a simple argument will allow us to finish the proof.

By Theorems 2.3 and 2.5, there exist a measurable function $\tau$ : $[0,+\infty) \rightarrow \overline{\mathbb{D}}$ and a Herglotz function $p(z, t)$ of order $d$ such that for all $z \in \mathbb{D}$ and all $s \geq 0$,

$$
\frac{\partial \psi_{s, t}(z)}{\partial t}=\left(\psi_{s, t}(z)-\tau(t)\right)\left(\overline{\tau(t)} \psi_{s, t}(z)-1\right) p\left(\psi_{s, t}(z), t\right) \text { a.e. } t \in[0,+\infty)
$$


Since $\psi_{s, t}(0)=0, \psi_{s, t}^{\prime}(0)>0, t \geq s \geq 0$, we conclude that $\tau(t) \equiv 0$. In this case, one can rewrite equation (3.1) in the form

$$
\frac{\partial \psi_{s, t}(z)}{\partial t}=-\psi_{s, t}(z) p\left(\psi_{s, t}(z), t\right) .
$$

We will show that the functions

$$
g_{s}(z):=\lim _{t \rightarrow+\infty} \frac{\psi_{s, t}(z)}{\psi_{0, t}^{\prime}(0)}
$$

where the limit is attained uniformly on compact subsets of the unit disk, form a Loewner chain associated with $\left(\psi_{s, t}\right)$. Our proof of the existence of that limit follows the approach given in [28, Chapter 6]. However, for the sake of clearness and completeness, we include the details.

Assume for a moment that such a limit does exist. Then $g_{s}^{\prime}(0):=$ $\lim _{t \rightarrow+\infty} \frac{\psi_{s, t}^{\prime}(0)}{\psi_{0, t}^{\prime}(0)}=\frac{1}{\psi_{0, s}^{\prime}(0)}>0$. Moreover, since all the functions $\psi_{s, t}$ are univalent [5, Corollary 6.3], we conclude that the function $g_{s}$ is univalent for all $s \geq 0$. Moreover, by construction

$$
g_{t} \circ \psi_{s, t}(z)=\lim _{u \rightarrow+\infty} \frac{\psi_{t, u}\left(\psi_{s, t}(z)\right)}{\psi_{0, u}^{\prime}(0)}=\lim _{u \rightarrow+\infty} \frac{\psi_{s, u}(z)}{\psi_{0, u}^{\prime}(0)}=g_{s}(z), \quad 0 \leq s \leq t<+\infty .
$$

Therefore, by Lemma 3.2, the family $\left(g_{t}\right)$ is a Loewner chain of order $d$ associated with $\left(\psi_{s, t}\right)$. Also, it is clear that it is a normalized Loewner chain.

Therefore, we have only to prove the existence of (3.3).

By [5, Proof of Theorem 7.1], for all $z \in \mathbb{D}$ and $t>s \geq 0$,

$$
\psi_{s, t}(z)=z \exp \left(-\int_{s}^{t} p\left(\psi_{s, \xi}(z), \xi\right) d \xi\right)
$$

Write $\Lambda_{s, t}(z):=\int_{s}^{t}\left(p(0, \xi)-p\left(\psi_{s, \xi}(z), \xi\right)\right) d \xi$. Notice that

$$
\psi_{s, t}^{\prime}(0)=\exp \left(-\int_{s}^{t} p(0, \xi) d \xi\right)>0 .
$$

Therefore,

$$
\frac{\psi_{s, t}(z)}{\psi_{0, t}^{\prime}(0)}=z \exp \left(\int_{0}^{s} p(0, \xi) d \xi\right) \exp \left(\Lambda_{s, t}(z)\right)
$$

Now in order to prove the existence of the limit (3.3), it is sufficient to show that $\Lambda_{s, t}$ has a limit as $t \rightarrow+\infty$ which is attained uniformly on compact subsets of the unit disk. 
By property EF2, we have that $\psi_{0, t}^{\prime}(0)=\psi_{s, t}^{\prime}(0) \psi_{0, s}^{\prime}(0) \leq \psi_{0, s}^{\prime}(0)$, because $\psi_{s, t}(0)=0$ and $\psi_{s, t}(\mathbb{D}) \subseteq \mathbb{D}$. That is

$$
\frac{\partial \psi_{0, t}^{\prime}(0)}{\partial t} \leq 0, \quad \text { a.e. } t \in[0,+\infty) .
$$

Since

$\frac{\partial \psi_{0, t}^{\prime}(0)}{\partial t}=-p(0, t) \exp \left(-\int_{s}^{t} p(0, \xi) d \xi\right)=-p(0, t) \psi_{s, t}^{\prime}(0), \quad$ a.e. $t \in[0,+\infty)$, we conclude that $p(0, t) \geq 0$ for a.e. $t \in[0, \infty)$.

When $\operatorname{Re} p(\cdot, \xi)>0$ (otherwise, $p(\cdot, \xi)$ is constant), necessarily $p(0, \xi)>0$ and the holomorphic map $z \mapsto \frac{p(z, \xi)-p(0, \xi)}{p(z, \xi)+\overline{p(0, \xi)}}$ sends the unit disk into itself and fixes the origin. Then

$$
\begin{aligned}
|p(z, \xi)-p(0, \xi)| & \leq|z||p(z, \xi)+\overline{p(0, \xi)}| \leq|z||p(z, \xi)|+|z||p(0, \xi)| \\
& \leq|z| \frac{1+|z|}{1-|z|}|p(0, \xi)|+|z||p(0, \xi)|=\frac{2|z|}{1-|z|} p(0, \xi),
\end{aligned}
$$

where we have used [28, pages 39-40]. Therefore, by [28, Theorem 1.6], we have

$$
\begin{aligned}
\left|p\left(\psi_{s, \xi}(z), \xi\right)-p(0, \xi)\right| & \leq \frac{2\left|\psi_{s, \xi}(z)\right|}{1-\left|\psi_{s, \xi}(z)\right|} p(0, \xi) \leq \frac{2\left|\psi_{s, \xi}(z)\right|}{1-|z|} p(0, \xi) \\
& \leq \frac{2\left|\psi_{s, \xi}^{\prime}(0)\right|}{(1-|z|)^{3}} p(0, \xi)=\frac{2 \exp \left(-\int_{s}^{\xi} p(0, u) d u\right)}{(1-|z|)^{3}} p(0, \xi) .
\end{aligned}
$$

Now, we can bound the function $\Lambda_{s, \cdot}(z)$ :

$$
\begin{aligned}
\mid \Lambda_{s, t}(z)- & \Lambda_{s, u}(z)\left|\leq \int_{u}^{t}\right| p(0, \xi)-p\left(\psi_{s, \xi}(z), \xi\right) \mid d \xi \\
& \leq \frac{2}{(1-|z|)^{3}} \int_{u}^{t} \exp \left(-\int_{0}^{\xi} p(0, u) d u\right) p(0, \xi) d \xi \\
& =\frac{2}{(1-|z|)^{3}} \int_{u}^{t} \frac{\partial}{\partial \xi}\left[-\exp \left(-\int_{0}^{\xi} p(0, u) d u\right)\right] d \xi \\
& =\frac{2}{(1-|z|)^{3}}\left[\exp \left(-\int_{0}^{u} p(0, \xi) d \xi\right)-\exp \left(-\int_{0}^{t} p(0, \xi) d \xi\right)\right] .
\end{aligned}
$$

Finally, from these last inequalities and the fact that

$$
\lim _{t \rightarrow+\infty} \exp \left(-\int_{0}^{t} p(0, \xi) d \xi\right) \in[0,1]
$$

(recall that $p(0, \xi) \geq 0$ for a.e. $\xi \in[0,+\infty)$ ), we conclude that the limit (3.3) does exist. 
Now we consider the family $f_{t}=g_{t} \circ h_{t}^{-1}$. It easy to see that $\left(f_{t}\right)$ satisfies the hypothesis of Lemma 3.2 and hence it is a Loewner chain of order $d$ associated with $\left(\varphi_{s, t}\right)$. Since $f_{0}=g_{0}$, the Loewner chain $\left(f_{t}\right)$ is normalized.

Now, let us describe the set $\Omega=\cup_{t \geq 0} f_{t}(\mathbb{D})=\cup_{t \geq 0} g_{t}(\mathbb{D})$. An easy computation shows $\psi_{0, t}^{\prime}(0)=\frac{\left|\varphi_{0, t}^{\prime}(0)\right|}{1-\left|\varphi_{0, t}(0)\right|^{2}}$. In particular, since the map $t \mapsto \psi_{0, t}^{\prime}(0)$ is monotone, the number

$$
\beta=\lim _{t \rightarrow+\infty} \frac{\left|\varphi_{0, t}^{\prime}(0)\right|}{1-\left|\varphi_{0, t}(0)\right|^{2}}
$$

is well-defined.

In view of the equality $g_{t}^{\prime}(0)=1 / \psi_{0, t}^{\prime}(0)$, Koebe's theorem shows that $g_{t}(\mathbb{D})$ contains a disk of radius $1 /\left(4 \psi_{0, t}^{\prime}(0)\right)$ centered at the origin. In particular, if $\beta=0$, then $\cup_{t>0} g_{t}(\mathbb{D})=\mathbb{C}$.

Suppose now that $\beta>0$. We have proved that in this case $\psi_{s, t}$ has a limit $\psi_{s}$ as $t \rightarrow+\infty$. Note that $\psi_{s}^{\prime}(0)=\beta / \psi_{0, s}^{\prime}(0) \rightarrow 1$ as $s \rightarrow+\infty$, while $\psi_{s}(\mathbb{D}) \subset \mathbb{D}$ and $\psi_{s}(0)=0$. It follows that $\psi_{s} \rightarrow \mathrm{id}_{\mathbb{D}}$ as $s \rightarrow+\infty$. Then $g_{s}$ tends to the mapping $z \mapsto z / \beta$ as $s \rightarrow+\infty$ locally uniformly in $\mathbb{D}$. Since $g_{s}(\mathbb{D})$ forms an increasing family of domains, it follows that $\cup_{s \geq 0} g_{s}(\mathbb{D})=\{z:|z|<1 / \beta\}$.

The proof is now finished.

In the above proof we have obtained that the function $\beta:[0,+\infty) \rightarrow$ $(0,1]$ given by

$$
\beta(t):=\frac{1}{1-\left|\varphi_{0, t}(0)\right|^{2}}\left|\varphi_{0, t}^{\prime}(0)\right| \quad \text { for all } t \geq 0, z \in \mathbb{D}
$$

is non-increasing and, as a consequence, the following limit exist

$$
\beta:=\lim _{t \rightarrow+\infty} \beta(t) \in[0,1] .
$$

This number will play a crucial role in the study of uniqueness of Loewner chains associated with the evolution family $\left(\varphi_{s, t}\right)$. For this reason, in the next proposition we analyze in full generality the above limit.

Proposition 3.4. Let $\left(\varphi_{s, t}\right)$ be an evolution family of order $d \in[1,+\infty]$ and define

$$
\beta_{z}(t):=\frac{1-|z|^{2}}{1-\left|\varphi_{0, t}(z)\right|^{2}}\left|\varphi_{0, t}^{\prime}(z)\right| \quad \text { for all } t \geq 0, z \in \mathbb{D} \text {. }
$$

Then

1. For all $z \in \mathbb{D}$, the map $\beta_{z}:[0,+\infty) \rightarrow(0,1]$ is absolutely continuous and non-increasing. In particular, there exists the following limit

$$
\beta(z):=\lim _{t \rightarrow+\infty} \beta_{z}(t) .
$$


2. The following assertions are equivalent:

(a) There exists $z \in \mathbb{D}$ such that $\beta(z)=0$.

(b) For all $z \in \mathbb{D}$ we have $\beta(z)=0$.

3. The following assertions are equivalent:

(a) There exists $z \in \mathbb{D}$ with $\beta(z)=1$.

(b) For all $z \in \mathbb{D}$, we have $\beta(z)=1$.

(c) For all $t \geq 0$, the map $\varphi_{0, t}$ is an automorphism.

(d) For all $0 \leq s \leq t$, the map $\varphi_{s, t}$ is an automorphism.

4. If there is $z \in \mathbb{D}$ such that $\beta(z)<1$, then there is $T \in[0,+\infty)$ such that $\varphi_{0, t}$ is an automorphism for all $0 \leq t \leq T$ and $\varphi_{0, t}$ is not an automorphism for all $t>T$.

Proof . By Proposition 2.9 we have $\varphi_{s, t}=h_{t} \circ \psi_{s, t} \circ h_{s}^{-1}$, where $\left(\psi_{s, t}\right)$ is an evolution family such that $\psi_{s, t}(0)=0$ and $\psi_{s, t}^{\prime}(0)>0$ for all $t \geq 0$, and $h_{t}$ is a conformal automorphism of $\mathbb{D}$ for each $t \geq 0$, with $h_{0}$ being the identity map.

One can check that

$$
\beta_{z}(t):=\frac{1-|z|^{2}}{1-\left|\varphi_{0, t}(z)\right|^{2}}\left|\varphi_{0, t}^{\prime}(z)\right|=\frac{1-|z|^{2}}{1-\left|\psi_{0, t}(z)\right|^{2}} \psi_{0, t}^{\prime}(z) \quad \text { for all } t \geq 0, z \in \mathbb{D} \text {. }
$$

Proof of (1). The absolute continuity of the function $\beta_{z}$ is just an easy consequence of Proposition 2.6.

Denote by $\widetilde{\rho}_{\mathbb{D}}$ the pseudo-hyperbolic distance in the unit disk. Since any holomorphic self-map of the unit disk is a contraction for $\widetilde{\rho}_{\mathbb{D}}$, given $s<t$ and $z, w \in \mathbb{D}$, we have

$$
\widetilde{\rho}_{D}\left(\varphi_{0, t}(w), \varphi_{0, t}(z)\right)=\widetilde{\rho}_{D}\left(\varphi_{s, t}\left(\varphi_{0, s}(w)\right), \varphi_{s, t}\left(\varphi_{0, s}(z)\right)\right) \leq \widetilde{\rho}_{D}\left(\varphi_{0, s}(w), \varphi_{0, s}(z)\right) .
$$

That is

$$
\left|\frac{\varphi_{0, t}(w)-\varphi_{0, t}(z)}{1-\overline{\varphi_{0, t}(w)} \varphi_{0, t}(z)}\right| \leq\left|\frac{\varphi_{0, s}(w)-\varphi_{0, s}(z)}{1-\overline{\varphi_{0, s}(w)} \varphi_{0, s}(z)}\right| .
$$

Dividing by $|w-z|(w \neq z)$ and taking limits as $w \rightarrow z$, we deduce that

$$
\frac{\left|\varphi_{0, t}^{\prime}(z)\right|}{1-\left|\varphi_{0, t}(z)\right|^{2}} \leq \frac{\left|\varphi_{0, s}^{\prime}(z)\right|}{1-\left|\varphi_{0, s}(z)\right|^{2}}
$$

Thus $\beta_{z}(t) \leq \beta_{z}(s)$ for all $0 \leq s<t<+\infty$. 
Proof of (2). Notice that we know that the number $\beta(0)=\lim _{t \rightarrow+\infty} \psi_{s, t}^{\prime}(0)$ is well defined. Moreover, the family of functions $\left(\psi_{0, t}\right)_{t \geq 0}$ is normal. So there is a sequence $\left(t_{n}\right) \rightarrow+\infty$ such that the limit $f(z)=\lim _{n} \psi_{0, t_{n}}(z)$ exists for all $z \in \mathbb{D}$ and it is attained uniformly on compact subsets of $\mathbb{D}$. The function $f$ is either constant or univalent in $\mathbb{D}$, with $f(0)=0$ and $f^{\prime}(0)=\beta(0)$. Therefore $f$ vanishes identically if and only if $\beta(0)=0$. Otherwise, $f$ is univalent and $f^{\prime}(z) \neq 0$ for all $z \in \mathbb{D}$. Now, observe that

$$
\begin{aligned}
\beta(z) & =\lim _{t \rightarrow+\infty} \beta_{z}(t)=\lim _{n \rightarrow+\infty} \beta_{z}\left(t_{n}\right) \\
& =\lim _{n \rightarrow+\infty} \frac{1-|z|^{2}}{1-\left|\psi_{0, t_{n}}(z)\right|^{2}}\left|\psi_{0, t_{n}}^{\prime}(z)\right|=\frac{1-|z|^{2}}{1-|f(z)|^{2}}\left|f^{\prime}(z)\right| .
\end{aligned}
$$

That is, $\beta(z)=0$ for some $z \in \mathbb{D}$ if and only if $f^{\prime}(z)=0$ for some $z \in \mathbb{D}$ if and only if $f$ is zero (recall that $f(0)=0$ ).

Assertions (3) and (4) are easy and we leave their proofs to the reader.

Definition 3.5. Let $\varphi_{s, t}$ be an evolution family and $\beta=\lim _{t \rightarrow+\infty} \frac{\left|\varphi_{0, t}^{\prime}(0)\right|}{1-\left|\varphi_{0, t}(0)\right|^{2}}$. Let $\left(f_{t}\right)$ be a normalized Loewner chain associated with $\varphi_{s, t}$. We say that $\left(f_{t}\right)$ is a standard Loewner chain if $\cup_{t \geq 0} f_{t}(\mathbb{D})=\{z:|z|<1 / \beta\}$ (obviously, when $\beta=0$, by $\{z:|z|<1 / \beta\}$ we mean the complex plane $\mathbb{C})$.

Note that if $\left(f_{t}\right)$ is a Loewner chain associated with a given evolution family $\left(\varphi_{s, t}\right)$ and $h$ is any univalent holomorphic function in $\Omega:=\cup_{t \geq 0} f_{t}(\mathbb{D})$, then the formula $g_{t}=h \circ f_{t}, t \geq 0$, defines a Loewner chain which is also associated with $\left(\varphi_{s, t}\right)$. In view of this remark, the following theorem gives a necessary and sufficient condition for an evolution family to have a unique normalized Loewner chain associated with it. Moreover, in case of non-uniqueness, the set of all normalized Loewner chains associated with $\left(\varphi_{s, t}\right)$ is explicitly described.

As usual, we denote by $\mathcal{S}$ the class of all univalent holomorphic functions $h$ in the unit disk $\mathbb{D}$, normalized by $h(0)=h^{\prime}(0)-1=0$. As above, $\beta=\lim _{t \rightarrow+\infty}\left|\varphi_{0, t}^{\prime}(0)\right| /\left(1-\left|\varphi_{0, t}(0)\right|^{2}\right)$.

Theorem 3.6. Let $\left(\varphi_{s, t}\right)$ be an evolution family.

1. There is a unique standard Loewner chain $\left(f_{t}\right)$ associated with $\left(\varphi_{s, t}\right)$.

2. If $\beta=0$, then there is a unique normalized Loewner chain $\left(f_{t}\right)$ associated with $\left(\varphi_{s, t}\right)$ (and obviously, it is the standard one.)

3. If $\beta>0$ and $\left(g_{t}\right)$ is a normalized Loewner chain associated with $\left(\varphi_{s, t}\right)$, then there is an $h \in \mathcal{S}$ such that

$$
g_{t}(z)=h\left(\beta f_{t}(z)\right) / \beta,
$$

where $\left(f_{t}\right)$ is the unique standard Loewner chain associated with $\left(\varphi_{s, t}\right)$. 
Proof. Let $\left(f_{t}\right)$ be the standard Loewner chain built in Theorem 3.3 and $\left(g_{t}\right)$ another normalized Loewner chain associated with the evolution family $\left(\varphi_{s, t}\right)$. For each $t \geq 0$ denote by $k_{t}: f_{t}(\mathbb{D}) \rightarrow g_{t}(\mathbb{D})$ the function $k_{t}=g_{t} \circ f_{t}^{-1}$. Write $\Omega_{1}=\cup_{t \geq 0} f_{t}(\mathbb{D})=\{z:|z|<1 / \beta\}$ and $\Omega_{2}=\cup_{t \geq 0} g_{t}(\mathbb{D})$.

If $s<t$ and $w \in f_{s}(\mathbb{D})$ with $w=f_{s}(z)$, we have that

$$
\begin{aligned}
k_{t}(w) & =g_{t} \circ f_{t}^{-1}\left(f_{s}(z)\right)=g_{t} \circ f_{t}^{-1}\left(f_{t}\left(\varphi_{s, t}(z)\right)\right) \\
& =g_{t}\left(\varphi_{s, t}(z)\right)=g_{s}(z)=g_{s}\left(f_{s}^{-1}(w)\right)=k_{s}(w) .
\end{aligned}
$$

That is, $k_{t \mid f_{s}(\mathbb{D})}=k_{s}$. This property says that the function $k: \Omega_{1} \rightarrow \Omega_{2}$ defined by $k(w):=k_{t}(w)$ for some (or any) $t$ such that $w \in f_{t}(\mathbb{D})$ is welldefined, univalent and onto. Moreover $k(0)=0$ and $k^{\prime}(0)=1$. Notice that $k \circ f_{t}=g_{t}$ for all $t$.

Now suppose that $\beta=0$. Then $\Omega_{1}=\mathbb{C}$. Since $\Omega_{2}$ is a simply connected domain biholomorphic to $\mathbb{C}$, we also have that $\Omega_{2}=\mathbb{C}$. In this case, $k$ is a univalent entire function such that $k(0)=k^{\prime}(0)-1=0$. Then $k$ is the identity and $f_{t}=g_{t}$ for all $t$. This implies statement (2) and statement (1) for the case $\beta=0$.

If $\beta>0$, denote by $h: \mathbb{D} \rightarrow \Omega_{2}$ the function $h(z)=\beta k(z / \beta)$. Obviously, $h$ belongs to $\mathcal{S}$ and satisfies (3.6). This proves statement (3). Finally, if $\left(g_{t}\right)$ is also a standard Loewner chain associated with $\left(\varphi_{s, t}\right)$, then $\Omega_{2}=\{z:|z|<1 / \beta\}$. In this case $k:\{z:|z|<1 / \beta\} \rightarrow\{z:|z|<1 / \beta\}$ is biholomorphic and $k(0)=k^{\prime}(0)-1=1$. That is $k$ is the identity and $f_{t}=g_{t}$ for all $t \geq 0$. This proves statement (1) for $\beta>0$.

The proof is now complete.

Remark 3.7. It is clear from the above proof that one can define the standard Loewner chain as the unique normalized Loewner chain $\left(f_{t}\right)$, associated with the evolution family $\left(\varphi_{s, t}\right)$, such that $\cup_{t \geq 0} f_{t}(\mathbb{D})$ is either an Euclidean disk or the whole complex plane.

Our next theorem says that, in some particular cases, the univalence of the functions which form a Loewner chain can be replaced by an appropriate bound of these functions on certain hyperbolic disks.

Theorem 3.8. Let $\left(\varphi_{s, t}\right)$ be an evolution family in the unit disk having a unique normalized Loewner chain associated with it. Suppose $\left(f_{t}\right)_{t \geq 0}$ is a family in $\operatorname{Hol}(\mathbb{D}, \mathbb{C})$. Then $\left(f_{t}\right)$ is the unique normalized Loewner chain associated with $\left(\varphi_{s, t}\right)$ if and only if the following three conditions are satisfied:

1. The function $f_{0}$ is normalized, that is, $f_{0}(0)=f_{0}^{\prime}(0)-1=0$.

2. The equation $f_{t} \circ \varphi_{s, t}=f_{s}$ holds for any $0 \leq s \leq t$. 
3. For each $R>0$, there exists some $C>0$ (independent of $t$ ) such that for all $t \geq 0$ the following inequality

$$
\left|f_{t}(z)\right| \leq \frac{C}{\beta(t)}
$$

where

$$
\beta(t)=\frac{\left|\varphi_{0, t}^{\prime}(0)\right|}{1-\left|\varphi_{0, t}(0)\right|^{2}}, \quad t \geq 0,
$$

holds for any $z$ in the hyperbolic disk of radius $R$ centered at $\varphi_{0, t}(0)$.

Proof. Before dealing with the proof of the theorem, we comment (really recall) some facts and notations which will be used later on and which have been shown in the course of proofs of previous results. In our case, according to Theorem 3.6, we know that $\lim _{t \rightarrow+\infty} \beta(t)=0$.

Write

$$
a(t)=\varphi_{0, t}(0), \quad b(t)=\frac{\varphi_{0, t}^{\prime}(0)}{\left|\varphi_{0, t}^{\prime}(0)\right|}, \quad h_{t}(z)=\frac{b(t) z+a(t)}{1+b(t) \overline{a(t)} z},
$$

and

$$
h_{t}^{-1}(z)=\overline{b(t)} \frac{z-a(t)}{1-\overline{a(t)} z},
$$

for all $z \in \mathbb{D}$ and all $t \geq 0$. Clearly, $a(0)=0$ and $b(0)=1$. Finally, define $\psi_{s, t}=h_{t}^{-1} \circ \varphi_{s, t} \circ h_{s}$ for all $0 \leq s \leq t$. One can easily prove that $\psi_{s, t}(0)=0$ and $\psi_{s, t}^{\prime}(0)=\beta(t) / \beta(s)>0$ for all $0 \leq s \leq t$. Hence, by Proposition 2.9, $\left(\psi_{s, t}\right)$ is an evolution family.

$(\Rightarrow)$ Assume that $\left(f_{t}\right)$ is the (unique) normalized Loewner chain associated with $\left(\varphi_{s, t}\right)$. By the very definition of normalized Loewner chains, we see that only property (3) requires a proof. Note that

$$
\frac{\left|\psi_{0, t}^{\prime}(0)\right|}{1-\left|\psi_{0, t}(0)\right|^{2}}=\left|\psi_{0, t}^{\prime}(0)\right|=\beta(t) \rightarrow 0 \quad \text { as } \quad t \rightarrow+\infty \text {. }
$$

Therefore, according to Theorem 3.6 (now applied to the evolution family $\left(\psi_{s, t}\right)$ ), we deduce that $\left(\psi_{s, t}\right)$ has also a unique normalized Loewner chain associated with it. Moreover, such a Loewner chain $\left(g_{t}\right)$ satisfies the equality $g_{t}^{\prime}(0) \psi_{s, t}^{\prime}(0)=g_{s}^{\prime}(0)$. Consequently, $g_{t}^{\prime}(0) \beta(t)=g_{s}^{\prime}(0) \beta(s)$ for all $t, s \geq 0$. But $g_{0}^{\prime}(0) \beta(0)=1$. Thus $g_{s}^{\prime}(0)=1 / \beta(s)$ for all $s \geq 0$. Using the Distortion Theorem, we conclude that

$$
\left|g_{s}(z)\right| \leq \frac{1}{\beta(s)} \frac{|z|}{(1-|z|)^{2}}
$$

for all $s \geq 0$ and for all $z \in \mathbb{D}$. 
Now, fix $R>0$ and $s \geq 0$ and consider $r=\frac{e^{R}-1}{e^{R}+1} \in(0,1)$. Take $z$ in the hyperbolic disk of radius $R$ centered at the point $a(s)=\varphi_{0, s}(0)$. We have that

$$
\left.\rho_{\mathbb{D}}\left(h_{s}^{-1}(z), 0\right)\right)=\rho_{\mathbb{D}}\left(h_{s}^{-1}(z), h_{s}^{-1}(a(s))\right)=\rho_{\mathbb{D}}(z, a(s)) \leq R .
$$

Thus, $\left|h_{s}^{-1}(z)\right| \leq r$ and

$$
\left|f_{s}(z)\right|=\left|g_{s}\left(h_{s}^{-1}(z)\right)\right| \leq \frac{1}{\beta(s)} \frac{r}{(1-r)^{2}}=\frac{e^{2 R}-1}{2 \beta(s)} .
$$

$(\Leftarrow)$ First of all, bearing in mind Lemma 3.2 and property $(1)$ combined with Theorem 3.6, we see that we only have to prove the univalence of each function $f_{t}$. We start by defining

$$
g_{t}:=f_{t} \circ h_{t} \in \operatorname{Hol}(\mathbb{D}, \mathbb{C}), t \geq 0 .
$$

By property (2), we observe that

$$
g_{t} \circ \psi_{s, t}=g_{s}, 0 \leq s \leq t .
$$

We notice that the family $\left(g_{t}\right)$ satisfies the following three properties:

(a) $g_{t}(0)=0$, for all $t \geq 0$.

(b) $g_{t}^{\prime}(0)=\beta(t)^{-1}$, for all $t \geq 0$.

(c) For all $R>0$, there exists some $C>0$ such that, for all $t \geq 0$ and all $|z| \leq R$, we have

$$
\left|g_{t}(z)\right| \leq C \beta(t)^{-1} .
$$

Now, fix $s \geq 0$ and $r \in(0,1)$ and suppose that $|z| \leq r$. Take also some $R \in(0,1)$ with $R>r$. By Schwarz Lemma,

$$
\left|\psi_{s, t}(z)\right| \leq|z| \leq r, \text { for all } t \geq s .
$$

Then by the Cauchy Integral Formula, for all $t \geq s$ we have

$$
\begin{aligned}
\left|g_{s}(z)-\beta(t)^{-1} \psi_{s, t}(z)\right| & =\left|g_{t}\left(\psi_{s, t}(z)\right)-\beta(t)^{-1} \psi_{s, t}(z)\right| \\
& =\left|g_{t}\left(\psi_{s, t}(z)\right)-g_{t}(0)-g_{t}^{\prime}(0) \psi_{s, t}(z)\right| \\
& =\left|\frac{1}{2 \pi i} \int_{C^{+}(0, R)} g_{t}(\xi) \frac{\left(\psi_{s, t}(z)\right)^{2}}{\xi^{2}\left(\xi-\psi_{s, t}(z)\right)} d \xi\right| \\
& \leq \frac{2 \pi R}{2 \pi R^{2}(R-r)}\left|\psi_{s, t}(z)\right|^{2} \max \left\{\left|g_{t}(\xi)\right|:|\xi| \leq R\right\} .
\end{aligned}
$$

Therefore, by property (3), we can find $C=C(R)$ (independent of $t$ ) such that

$$
\left|g_{s}(z)-\beta(t)^{-1} \psi_{s, t}(z)\right| \leq \frac{C}{R(R-r)} \beta(t)^{-1}\left|\psi_{s, t}(z)\right|^{2} .
$$


In fact, since $\lim _{t \rightarrow+\infty} \beta(t)=0$ and by the Distortion Theorem, we deduce that

$$
\begin{aligned}
\left|g_{s}(z)-\beta(t)^{-1} \psi_{s, t}(z)\right| & \leq \frac{C}{R(R-r)} \beta(t)^{-1}\left|\psi_{s, t}^{\prime}(0)\right|^{2}\left(\frac{r}{(1-r)^{2}}\right)^{2} \\
& \leq \frac{C r^{2}}{R(R-r)(1-r)^{4}} \frac{1}{\beta(t)} \frac{\beta^{2}(t)}{\beta^{2}(s)} \\
& =\frac{C r^{2}}{R(R-r)(1-r)^{4} \beta^{2}(s)} \beta(t) \rightarrow 0 \quad \text { as } \quad t \rightarrow+\infty .
\end{aligned}
$$

Therefore, we conclude that

$$
g_{s}=\lim _{t \rightarrow+\infty} \beta(t)^{-1} \psi_{s, t},
$$

in the compact-open topology of $\operatorname{Hol}(\mathbb{D}, \mathbb{C})$. By Hurwitz's Theorem and property (b), we find that $g_{s}$ is univalent. Since $h_{t}$ is an automorphism of the disk, we finally conclude that $f_{s}$ is univalent as well. The proof is complete.

Remark 3.9. The above proof shows that statement (3) in this last theorem can be replaced by "for all $z \in \mathbb{D}$ and for all $s \geq 0$, the following inequality holds

$$
\left|f_{s} \circ h_{s}(z)\right| \leq \frac{1}{\beta(s)} \frac{|z|}{(1-|z|)^{2}},
$$

where, as usual, $h_{s}(z)=\frac{b(s) z+a(s)}{1+b(s) \overline{a(s) z}}, a(t)=\varphi_{0, a}(0)$, and $b(s)=\frac{\varphi_{0, s}^{\prime}(0)}{\left|\varphi_{0, s}^{\prime}(0)\right|}$."

\section{Loewner chains and partial differential equations}

In classical Loewner theory any Loewner chain satisfies the LoewnerKufarev PDE, while the corresponding evolution family satisfies the Loewner-Kufarev ODE with the same driving term. Now we prove an analogue of this statement in our general setting.

Theorem 4.1. The following assertions hold.

1. Let $\left(f_{t}\right)$ be a Loewner chain of order $d \in[1,+\infty]$. Then

(a) There exists a set $N \subset[0,+\infty)$ (not depending on $z)$ of zero measure such that for every $s \in(0,+\infty) \backslash N$ the function

$$
z \in \mathbb{D} \mapsto \frac{\partial f_{s}(z)}{\partial s}:=\lim _{h \rightarrow 0} \frac{f_{s+h}(z)-f_{s}(z)}{h} \in \mathbb{C}
$$

is a well-defined holomorphic function on $\mathbb{D}$. 
(b) There exist a Herglotz vector field $G$ of order $d$ and a set $N \subset$ $[0,+\infty)$ (not depending on $z$ ) of zero measure such that for every $s \in(0,+\infty) \backslash N$ and every $z \in \mathbb{D}$,

$$
\frac{\partial f_{s}(z)}{\partial s}=-G(z, s) f_{s}^{\prime}(z)
$$

2. Let $G$ be a Herglotz vector field of order $d \in[1,+\infty]$ associated with the evolution family $\left(\varphi_{s, t}\right)$. Suppose that $\left(f_{t}\right)$ is a family of univalent holomorphic functions in the unit disk such that for each $z$, the function $t \mapsto f_{t}(z)$ is absolutely continuous and

$$
\frac{\partial f_{s}(z)}{\partial s}=-G(z, s) f_{s}^{\prime}(z) \quad \text { for every } z \in \mathbb{D} \text {, a.e. } s \in[0,+\infty) .
$$

Then $\left(f_{t}\right)$ is a Loewner chain of order $d$ associated with the evolution family $\left(\varphi_{s, t}\right)$.

Proof of (1.a). By the very definition of Loewner chain, the map $s \in$ $[0,+\infty) \mapsto f_{s}(z) \in \mathbb{C}$ is absolutely continuous, for all fixed $z \in \mathbb{D}$. Thus there exists a set of zero measure $N_{1}(z) \subset[0,+\infty)$ such that for every $s \in[0,+\infty) \backslash N_{1}(z)$ the following limit exists

$$
D_{s}(z)=\frac{\partial f_{s}(z)}{\partial s}=\lim _{h \rightarrow 0} \frac{f_{s+h}(z)-f_{s}(z)}{h} .
$$

Let $k_{n} \in L_{l o c}^{d}([0,+\infty), \mathbb{R})$ be a non negative function such that

$$
\left|f_{s}(z)-f_{t}(z)\right| \leq \int_{s}^{t} k_{n}(\xi) d \xi
$$

whenever $|z| \leq 1-1 / n$ and $0 \leq s \leq t$. For each $n$, there exists a set $N_{2}(n) \subset[0,+\infty)$ of zero measure such that for every $s \in[0,+\infty) \backslash N_{2}(t)$ there exists the limit

$$
k_{n}(s)=\lim _{h \rightarrow 0} \frac{1}{h} \int_{s}^{s+h} k_{n}(\eta) d \eta .
$$

Let us define

$$
N:=\left(\bigcup_{n=1}^{\infty} N_{1}\left(\frac{1}{n+1}\right)\right) \bigcup\left(\bigcup_{n=1}^{\infty} N_{2}(n)\right) .
$$

Obviously, $N$ is a subset of $[0,+\infty)$ of zero measure, independent of $z$. We are going to prove that for all $s \in[0,+\infty) \backslash N$ the following limit

$$
\lim _{h \rightarrow 0} \frac{f_{s+h}(z)-f_{s}(z)}{h}
$$

exists and attained uniformly on compact subsets of $\mathbb{D}$. 
First of all we show that for every $s \in(0,+\infty) \backslash N$ the family

$$
\mathcal{F}_{s}:=\left\{F_{h}:=\frac{1}{h}\left(f_{s+h}-f_{s}\right): 0<h<1 \text { or }-s<h<0\right\}
$$

is a relatively compact set in $\operatorname{Hol}(\mathbb{D}, \mathbb{C})$. To this aim, we consider two cases: (a) $0<h<1$; (b) $-s<h<0$.

Case $(a)$ : Fix $r \in(0,1)$. Let $n \in \mathbb{N}$ be such that $r<1-1 / n$. Then, for every $|z| \leq r$,

$$
\left|F_{h}(z)\right|=\left|\frac{1}{h}\left(f_{s+h}(z)-f_{s}(z)\right)\right| \leq \frac{1}{h} \int_{s}^{s+h} k_{n}(\xi) d \xi \leq \tilde{C}<+\infty,
$$

where the last inequality takes place since $s \notin N_{2}(n)$. Hence,

$$
\sup \left\{\left|F_{h}(z)\right|:|z| \leq r, 0<h<1\right\}<+\infty
$$

and consequently, by the Montel criterion, the subfamily of $\mathcal{F}_{s}$ with $0<h<$ 1 is a normal family in $\mathbb{D}$, as wanted.

Case $(b)$ : the proof is similar to that of case $(a)$ and we omit it.

Thus the family $\mathcal{F}_{s}$ is relatively compact in $\operatorname{Hol}(\mathbb{D}, \mathbb{C})$. Let $\psi, \phi$ be any pair of limit functions of $\mathcal{F}_{s}$ as $h \rightarrow 0$. By the very definition of $N$,

$$
D_{s}\left(\frac{1}{m+1}\right)=\psi\left(\frac{1}{m+1}\right)=\phi\left(\frac{1}{m+1}\right) \text {, }
$$

for every $m \in \mathbb{N}$. But $\left\{\frac{1}{m+1}\right\}$ is a sequence accumulating at 0 , hence by the identity principle $\psi=\phi$. This shows that

$$
\lim _{h \rightarrow 0} \frac{f_{s+h}(z)-f_{s}(z)}{h},
$$

exists for all $s \in(0,+\infty) \backslash N$ and is attained uniformly on compact subsets of $\mathbb{D}$, which finishes the proof of (1.a).

Proof of (1.b). By Theorem 3.1, there is an evolution family $\left(\varphi_{s, t}\right)$ of order $d$ associated with $\left(f_{t}\right)$. Let $G: \mathbb{D} \times[0,+\infty) \rightarrow \mathbb{C}$ be the Herglotz vector field whose positive trajectories are $\left(\varphi_{s, t}\right)$ (such a vector field exists by Theorem 2.3). Let $N_{1} \subset[0,+\infty)$ be the set of zero measure given by [5, Theorem 6.4] such that $\frac{\partial \varphi_{0, u}}{\partial u}(z)=G\left(\varphi_{0, u}(z), u\right)$ for all $u \in(0,+\infty) \backslash N_{1}$ and all $z \in \mathbb{D}$. Let $N_{2} \subset[0,+\infty)$ stand for the set of zero measure which has been denoted by $N$ in part (1.a) of this theorem.

Let $N:=N_{1} \cup N_{2}$. Differentiating with respect to $t$ the equality $f_{t}\left(\varphi_{0, t}(z)\right)=f_{0}(z)$, for $z \in \mathbb{D}$ and $t \in(0,+\infty) \backslash N$ we obtain $0=f_{t}^{\prime}\left(\varphi_{0, t}(z)\right) \frac{\partial \varphi_{0, t}}{\partial t}(z)+\frac{\partial f_{t}}{\partial t}\left(\varphi_{0, t}(z)\right)=f_{t}^{\prime}\left(\varphi_{0, t}(z)\right) G\left(\varphi_{0, t}(z), t\right)+\frac{\partial f_{t}}{\partial t}\left(\varphi_{0, t}(z)\right)$. 
Therefore, $f_{t}^{\prime}(w) G(w, t)=-\frac{\partial f_{t}}{\partial t}(w)$ for all $w \in \varphi_{0, t}(\mathbb{D})$. Since the $\varphi_{0, t}$ 's are univalent, the identity principle for holomorphic maps implies that this equality is valid for the whole unit disk $\mathbb{D}$.

Proof of (2). Fix a point $z$ in the unit disk. Then, up to a set of measure zero, we have

$$
\begin{aligned}
\frac{\partial}{\partial t}\left(f_{t}\left(\varphi_{s, t}(z)\right)\right) & =f_{t}^{\prime}\left(\varphi_{s, t}(z)\right) \frac{\partial \varphi_{s, t}}{\partial t}(z)+\frac{\partial f_{t}}{\partial t}\left(\varphi_{s, t}(z)\right) \\
& =f_{t}^{\prime}\left(\varphi_{s, t}(z)\right) \frac{\partial \varphi_{s, t}}{\partial t}(z)-G\left(\varphi_{s, t}(z), t\right) f_{s}^{\prime}\left(\varphi_{s, t}(z)\right) \\
& =f_{t}^{\prime}\left(\varphi_{s, t}(z)\right)\left[\frac{\partial \varphi_{s, t}}{\partial t}(z)-G\left(\varphi_{s, t}(z), t\right)\right]=0 .
\end{aligned}
$$

Therefore, $f_{t}\left(\varphi_{s, t}(z)\right)$ does not depend on $t$. Hence, $f_{t}\left(\varphi_{s, t}(z)\right)=f_{s}\left(\varphi_{s, s}(z)\right)=$ $f_{s}(z)$ and the proof finishes just by applying Lemma 3.2.

\section{Remarks about semigroups}

A (one-parameter) semigroup of holomorphic functions is a continuous homomorphism $\Phi: t \mapsto \Phi(t)=\phi_{t}$ from the additive semigroup of non-negative real numbers into the composition semigroup of holomorphic self-maps of $\mathbb{D}$. Namely, $\Phi$ satisfies the following three conditions:

S1. $\phi_{0}$ is the identity in $\mathbb{D}$,

S2. $\phi_{t+s}=\phi_{t} \circ \phi_{s}$, for all $t, s \geq 0$,

S3. $\phi_{t}(z)$ tends to $z$ as $t$ tends to 0 , uniformly on compact subsets of $\mathbb{D}$.

Let $\left(\phi_{t}\right)$ be a semigroup of holomorphic self-maps of $\mathbb{D}$. Let $\varphi_{s, t}:=\phi_{t-s}$ for $0 \leq s \leq t<+\infty$. Then, by [5, Example 3.4], $\left(\varphi_{s, t}\right)$ is an evolution family of order $\infty$.

Given a semigroup $\Phi=\left(\phi_{t}\right)$, it is well-known (see [33], [4]) that there exists a unique holomorphic function $G: \mathbb{D} \rightarrow \mathbb{C}$ such that,

$$
\frac{\partial \phi_{t}(z)}{\partial t}=G\left(\phi_{t}(z)\right)=G(z) \frac{\partial \phi_{t}(z)}{\partial z} \text { for all } z \in \mathbb{D} \text { and } t \geq 0 .
$$

The function $G$ is known as the infinitesimal generator of the semigroup and, obviously, $G$ (that clearly does not depend on $t$ ) is the Herglotz vector field associated with the evolution family $\left(\varphi_{s, t}\right)$. Berkson and Porta [4] proved that there exist $\tau \in \overline{\mathbb{D}}$ and a holomorphic function $p: \mathbb{D} \rightarrow \mathbb{C}$ with $\operatorname{Re} p(z) \geq 0$ such that

$$
G(z)=(\tau-z)(1-\bar{\tau} z) p(z), \quad z \in \mathbb{D}
$$

and moreover, any function $G$ of this form is the infinitesimal generator of some semigroup. 
In this very particular case when the evolution family is generated by a semigroup, the point $\tau$ has a dynamical meaning. To explain this meaning, we have to recall some notions from iteration theory.

It can be easily deduced from the Schwarz-Pick lemma that a nonidentity self-map $\phi$ of the unit disk can have at most one fixed point in $\mathbb{D}$. If such a unique fixed point in $\mathbb{D}$ exists, it is usually called the Denjoy-Wolff point. The sequence of iterates $\left\{\phi_{n}\right\}$ of $\phi$ converges to it uniformly on the compact subsets of $\mathbb{D}$ whenever $\phi$ is not a disk automorphism.

If $\phi$ has no fixed points in $\mathbb{D}$, the Denjoy-Wolff theorem (see, e.g., [1]) guarantees the existence of a unique point $\tau$ on the unit circle $\partial \mathbb{D}$ which is the attractive fixed point, that is, the sequence of iterates $\left\{\phi_{n}\right\}$ converges to $\tau$ uniformly on the compact subsets of $\mathbb{D}$. Such a point $\tau$ is again called the Denjoy-Wolff point of $\phi$. When $\tau \in \partial \mathbb{D}$ is the Denjoy-Wolff point of $\phi$, the angular derivative $\phi^{\prime}(\tau)$ is actually real-valued and, moreover, $0<\phi^{\prime}(\tau) \leq 1$ (see [29]). As it is often done in the literature, we classify the holomorphic self-maps of the disk into three categories according to their behavior near the Denjoy-Wolff point:

(a) elliptic: the ones with a fixed point inside the unit disk $\mathbb{D}$;

(b) hyperbolic: the ones with the Denjoy-Wolff point $\tau \in \partial \mathbb{D}$ such that $\phi^{\prime}(\tau)<1$

(c) parabolic: the ones with the Denjoy-Wolff point $\tau \in \partial \mathbb{D}$ such that $\phi^{\prime}(\tau)=1$.

Going back to semigroups, we have to say that the point $\tau$ that appears in the Berkson-Porta representation formula for the infinitesimal generator of the semigroup $\left(\phi_{t}\right)$ is the Denjoy-Wolff point of all the functions $\phi_{t}$. In particular, all the functions share the Denjoy-Wolff point. But something more can be said. If there is $t_{0}>0$ such that the function $\phi_{t_{0}}$ is elliptic (resp. hyperbolic, parabolic) then all the functions of the semigroup are elliptic (resp. hyperbolic, parabolic).

Besides the above classification of self-maps of the unit disk, there are two quite different types of parabolic functions. To distinguish such functions, we have to recall the notion of hyperbolic step. Given a holomorphic self-map $\phi$ of $\mathbb{D}$ and a point $z_{0}$ in $\mathbb{D}$, we define the forward orbit of $z_{0}$ under $\phi$ as the sequence $z_{n}=\phi_{n}\left(z_{0}\right)$. It is customary to say that $\phi$ is of zero hyperbolic step if for some point $z_{0}$ the orbit $z_{n}=\phi_{n}\left(z_{0}\right)$ satisfies the condition $\lim _{n \rightarrow \infty} \rho_{\mathbb{D}}\left(z_{n}, z_{n+1}\right)=0$. It is well-known that the word "some" here can be replaced by "all". In other words, the definition does not depend on the choice of the initial point of the orbit (see, for example, [8]).

Using the Schwarz-Pick Lemma, it is easy to see that the maps which are not of zero hyperbolic step are precisely those holomorphic self-maps $\phi$ of $\mathbb{D}$ for which

$$
\lim _{n \rightarrow \infty} \rho_{\mathbb{D}}\left(z_{n}, z_{n+1}\right)>0
$$


for some forward orbit $\left\{z_{n}\right\}_{n=1}^{\infty}$ of $\phi$, and hence for all such orbits. This is the reason why they are called maps of positive hyperbolic step. For a survey of these properties, the reader may consult [8].

It is easy to show that if $\phi$ is elliptic and is not an automorphism, then it is of zero hyperbolic step. If $\phi$ is hyperbolic, then it is of positive hyperbolic step. For parabolic maps the situation is more complicated: there are parabolic functions of zero hyperbolic step and of positive hyperbolic step. For example, the following dichotomy holds for parabolic linear-fractional maps: every parabolic automorphism of $\mathbb{D}$ is of positive hyperbolic step, while all non-automorphic linear-fractional parabolic self-maps of $\mathbb{D}$ are of zero hyperbolic step. For semigroups of holomorphic functions we can state the following

Lemma 5.1. Let $\left(\phi_{t}\right)$ be a semigroup of parabolic functions in the unit disk. If there exists $t_{0}>0$ such that the function $\phi_{t_{0}}$ is of zero hyperbolic step, then all the functions $\phi_{t}$, with $t>0$, of the semigroup are of zero hyperbolic step.

Proof. In this proof we will use different well-known properties of the hyperbolic distance on simply connected domains in the complex plane that can be seen in [32].

By [35], there exists a univalent function $h: \mathbb{D} \rightarrow \mathbb{C}$, with $h(0)=0$, such that $h \circ \phi_{t}=h+t$ for all $t>0$. Write $\Omega=h(\mathbb{D})$ and denote by $\delta_{\Omega}(w)$ the Euclidean distance from $w \in \Omega$ to $\partial \Omega$. Since $\Omega+t \subseteq \Omega$ for all $t>0$, we can easily obtain that the function $\delta_{\Omega}:[0,+\infty) \rightarrow \mathbb{R}$ is non-decreasing (we are considering here the restriction of $\delta_{\Omega}$ to the half-line $[0,+\infty)$ ). By hypothesis, the sequence $\rho_{\mathbb{D}}\left(\phi_{n t_{0}}(0), \phi_{(n+1) t_{0}}(0)\right)$ goes to zero. Moreover, by the Distance Lemma, we have that

$$
\begin{aligned}
\rho_{\mathbb{D}}\left(\phi_{n t_{0}}(0), \phi_{(n+1) t_{0}}(0)\right) & =\rho_{\Omega}\left(h(0)+n t_{0}, h(0)+(n+1) t_{0}\right) \\
& =\rho_{\Omega}\left(n t_{0},(n+1) t_{0}\right) \\
& \geq \frac{1}{2} \log \left(1+\frac{\left|t_{0}\right|}{\min \left\{\delta_{\Omega}\left(n t_{0}\right), \delta_{\Omega}\left((n+1) t_{0}\right)\right\}}\right),
\end{aligned}
$$

where $\rho_{\Omega}$ denotes the hyperbolic distance on $\Omega$. Thus $\delta_{\Omega}\left((n+1) t_{0}\right)$ goes to $\infty$ and we conclude that $\lim _{t \rightarrow+\infty} \delta_{\Omega}(t)=\infty$.

Now fix $t>0$. Write $\Gamma_{n}=[n t,(n+1) t]$ and denote by $l_{\Omega}\left(\Gamma_{n}\right)$ the hyperbolic length of $\Gamma_{n}$ in $\Omega$. We have

$$
\rho_{\mathbb{D}}\left(\phi_{n t}(0), \phi_{(n+1) t}(0)\right)=\rho_{\Omega}(n t,(n+1) t) \leq l_{\Omega}\left(\Gamma_{n}\right) \leq 2 \int_{\Gamma_{n}} \frac{|d w|}{\delta_{\Omega}(w)} \leq 2 \frac{t}{\delta_{\Omega}(n t)},
$$

where again we have used the monotonicity of $\delta_{\Omega}$ on $[0,+\infty)$. Since the sequence $\delta_{\Omega}((n+1) t)$ goes to $\infty$, the above inequality implies that $\rho_{\mathbb{D}}\left(\phi_{n t}(0), \phi_{(n+1) t}(0)\right)$ tends to zero as $n$ goes to $\infty$. The arbitrariness of $t$ concludes the proof. 
What can we say about the Loewner chains associated with the evolution families $\left(\varphi_{s, t}\right)=\left(\phi_{t-s}\right)$ ?

If the semigroup $\left(\phi_{t}\right)$ is elliptic and its Denjoy-Wolff point is zero, then (see [35]) there is a complex number $c$ and a univalent function $h$ such that $\operatorname{Re} c \geq 0, h(0)=0, h^{\prime}(0)=1$, and

$$
h \circ \phi_{t}=e^{-c t} h .
$$

The function $h$ is called the Kœenigs function of the semigroup $\left(\phi_{t}\right)$. From equation (5.1), it is clear that the functions $f_{t}=e^{c t} h$ form a normalized Loewner chain associated with the evolution family $\left(\varphi_{s, t}\right)=\left(\phi_{t-s}\right)$. If $\operatorname{Re} c>0$, then $\cup_{t \geq 0} f_{t}(\mathbb{D})=\mathbb{C}$ and, by Theorem 3.6, this is the unique normalized Loewner chain associated with $\left(\varphi_{s, t}\right)$. In particular, this implies the uniqueness of the Kœnigs function, a fact which is very well-known. If $\operatorname{Re} c=0$, then $h$ is the identity map.

Now suppose that the Denjoy-Wolff point of the semigroup is on the boundary of the unit disk. Without loss of generality, we assume that such a point is 1 . Then there is a univalent function $h$ such that $h(0)=0$ and $h \circ \phi_{t}=$ $h+t$ [35]. As in the elliptic case, the function $h$ is referred to as the Kœnigs function of the semigroup $\left(\phi_{t}\right)$. Siskakis [34] (see also [35]) proved that the Kœnigs function is unique in this case as well. As an easy application of our results we will reprove the uniqueness of the Kœnigs function. Similarly to the elliptic case, we have that the functions $f_{t}=h-t$ form a Loewner chain associated with the evolution family $\left(\varphi_{s, t}\right)=\left(\phi_{t-s}\right)$. Notice that this Loewner chain is not necessarily normalized. To proceed let us distinguish the different type of semigroups.

If the functions $\phi_{t}$ are hyperbolic, then by [7, Theorem 2.1], there is a horizontal strip $\Omega$ such that the range of $h$ is included in $\Omega$ and $\cup_{t \geq 0} f_{t}(\mathbb{D})=\Omega$. In this case, there are much more Loewner chains associated with $\left(\varphi_{s, t}\right)$ but there is no other Loewner chain $\left(g_{t}\right)$ of the form $g_{t}=k-t$, where $k, k(0)=0$, is a univalent holomorphic function in $\mathbb{D}$. Indeed, if such another function $k$ does exist, then by Theorem 3.6, there is a univalent holomorphic function $a: \Omega \rightarrow \mathbb{C}$ such that $a(h(z)-t)=k(z)-t$ for all $t \geq 0$ and for all $z \in \mathbb{D}$. Derivating with respect to $t$, we have $a^{\prime}(h(z)-t)=1$ for all $t \geq 0$ and for all $z \in \mathbb{D}$. That is $a(z)=z+c$ for some constant $c$. Therefore $h(z)-t+c=k(z)-t$ for all $t \geq 0$ and for all $z \in \mathbb{D}$. Since $h(0)=k(0)=0$, we deduce that $c=0$ and $h=k$.

Consider now the parabolic case. According to the above lemma we have to distinguish two subcases. On one hand, if for some (or for any) $t_{0}>0$ the function $\phi_{t_{0}}$ is of zero hyperbolic step, then by [9, Theorem 3.1 and Proposition 3.3], the range of $h$ is not included in any horizontal half-plane. In this case, we have that $\cup_{t \geq 0} f_{t}(\mathbb{D})=\mathbb{C}$. Therefore, up to normalization, 
this is a unique Loewner chain associated with $\left(\varphi_{s, t}\right)$. On the other hand, if one (and then all) of the mappings $\phi_{t}$ is of positive hyperbolic step, then the range of $h$ is included in a horizontal half-plane $\Omega$. In fact, we can choose the half-plane such that $\cup_{t \geq 0} f_{t}(\mathbb{D})=\Omega$. By the same reason as in the hyperbolic case, there are much more Loewner chains associated with $\left(\varphi_{s, t}\right)$ but there is no other Loewner chains $\left(g_{t}\right)$ of the form $g_{t}=k-t$, where $k, k(0)=0$, is a univalent holomorphic function in $\mathbb{D}$. That is, again, the Kœnigs function of the semigroup is unique.

Acknowledgement. We also want to thank the anonymous referee for her/his precise suggestions and remarks.

\section{References}

[1] AвAтE, M.: Iteration theory of holomorphic maps on taut manifolds. Mediterranean Press, Rende, 1989.

[2] Aleksandrov, I. A.: Parametric continuations in the theory of univalent functions. Izdat. "Nauka", Moscow, 1976.

[3] BAuer, R. O.: Chordal Loewner families and univalent Cauchy transforms. J. Math. Anal. Appl. 302 (2005), no. 2, 484-501.

[4] Berkson, E. And Porta, H.: Semigroups of analytic functions and composition operators. Michigan Math. J. 25 (1978), no. 1, 101-115.

[5] Bracci, F., Contreras, M. D. and Díaz-Madrigal, S.: Evolution families and the Loewner equation I: the unit disk. To appear in J. Reine Angew. Math.

[6] Bracci, F., Contreras, M. D. and Díaz-Madrigal, S.: Evolution families and the Loewner equation II: complex hyperbolic manifolds. Math. Ann. 344 (2009), no. 4, 947-962.

[7] Contreras, M. D. And Díaz-Madrigal, S.: Analytic flows on the unit disk: angular derivatives and boundary fixed points. Pacific J. Math. 222 (2005), no. 2, 253-286.

[8] Contreras, M. D., Díaz-Madrigal, S. and Pommerenke, Ch.: Iteration in the unit disk: the parabolic zoo. In Complex and harmonic analysis, 63-91. Destech Publ., Lancaster, PA, 2007.

[9] Contreras, M. D., Díaz-Madrigal, S. and Pommerenke, Ch.: Some remarks on the Abel equation in the unit disk. J. Lond. Math. Soc. (2) $\mathbf{7 5}$ (2007), no. 3, 623-634.

[10] Conway, J. B.: Functions of one complex variable. Graduate Texts in Mathematics 11. Springer-Verlag, New York-Berlin, 1978.

[11] Conway, J. B.: Functions of one complex variable. II. Graduate Texts in Mathematics 159. Springer-Verlag, New York-Berlin, 1995.

[12] Duren, P. L.: Univalent Functions. Grundlehren der Mathematischen Wissenschaften 259. Springer-Verlag, New York, 1983. 
[13] Goluzin, G. M.: Geometric theory of functions of a complex variable. Translations of Mathematical Monographs 26. American Mathematical Society, Providence, RI, 1969.

[14] Goryainov, V.V.: Semigroups of conformal mappings. Math. USSR Sbornik 57 (1987), 463-483.

[15] Goryainov, V.V.: The Königs function and fractional integration of probability-generating functions. Mat. Sb. 184 (1993), no. 5, 55-74; translation in Russian Acad. Sci. Sb. Math. 79 (1994), no. 1, 47-61.

[16] Goryainov, V. V.: The embedding of iterations of probability-generating functions into continuous semigroups. Dokl. Akad. Nauk 330 (1993), no. 5, 539-541; trans. Russian Acad. Sci. Dokl. Math. 47 (1993), no. 3, 554-557.

[17] Goryainov, V. V. And BA, I.: Semigroups of conformal mappings of the upper half-plane into itself with hydrodynamic normalization at infinity. Ukrainian Math. J. 44 (1992), no. 10, 1209-1217.

[18] Kufarev, P.P.: On one-parameter families of analytic functions. Rec. Math. [Mat. Sbornik] N.S. 13 (55) (1943), 87-118.

[19] Lind, J. R.: A sharp condition for the Loewner equation to generate slits. Ann. Acad. Sci. Fenn. Math. 30 (2005), no. 1, 143-158.

[20] LAwler, G.F.: An introduction to the stochastic Loewner evolution. In Random walks and geometry, 261-293. Walter de Gruyter, Berlin, 2004.

[21] Lawler, G. F., Schramm, O. and Werner, W.: Values of Brownian intersection exponents. I. Half-plane exponents. Acta Math. 187 (2001), no. 2, 237-273.

[22] Lawler, G.F., Schramm, O. and Werner, W.: Values of Brownian intersection exponents. II. Plane exponents. Acta Math. 187 (2001), no. $2,275-308$.

[23] Lawler, G. F., Schramm, O. and Werner, W.: Values of Brownian intersection exponents. III. Two-sided exponents. Ann. Inst. H. Poincaré Probab. Statist. 38 (2002), no. 1, 109-123.

[24] Lawler, G.F., Schramm, O. and Werner, W.: Conformal invariance of planar loop-erased random walks and uniform spanning trees. Ann. Probab. 32 (2004), no. 1B, 939-995.

[25] Löwner, K.: Untersuchungen über schlichte konforme Abbildungen des Einheitskreises. Math. Ann. 89 (1923), no. 1-2, 103-121.

[26] Marshall, D. E. And Rohde, S.: The Loewner differential equation and slit mappings. J. Amer. Math. Soc. 18 (2005), no. 4, 763-778.

[27] Pommerenke, Ch.: Über die subordination analytischer funktionen. J. Reine Angew Math. 218 (1965), 159-173.

[28] Pommerenke, Ch.: Univalent Functions. Studia Mathematica/ Mathematische Lehrbücher, Band XXV. Vandenhoeck \& Ruprecht, Göttingen, 1975.

[29] Pommerenke, Ch.: Boundary behaviour of conformal maps. Grundlehren der Mathematischen Wissenschaften 299. Springer-Verlag, Berlin, 1992. 
[30] Prokhorov, D. And Vasil'ev, A.: Singular and tangent split solutions to the Löwner equation. In Analysis and Mathematical Physics, 455-463. Trends in Mathematics. Birkhäuser Verlag, 2009.

[31] Schramm, O.: Scaling limits of loop-erased random walks and uniform spanning trees. Israel J. Math. 118 (2000), 221-288.

[32] Shapiro, J. H.: Composition operators and classical function theory. Universitext: Tracts in Mathematics. Springer-Verlag, New York, 1993.

[33] Shoikhet, D.: Semigroups in geometrical function theory. Kluwer Academic Publishers, Dordrecht, 2001.

[34] Siskakis, A. G.: Semigroups of composition operators and the Cesaro operator on $H^{p}(D)$. Ph. D. Thesis, University of Illinois, 1985.

[35] Siskakis, A. G.: Semigroups of composition operators on spaces of analytic functions, a review. In Studies on composition operators (Laramie, 1996), 229-252. Contemp. Math. 213. Amer. Math. Soc., Providence, RI, 1998.

Recibido: 23 de diciembre de 2008

Manuel D. Contreras

Departamento de Matemática Aplicada II Escuela Técnica Superior de Ingenieros, Universidad de Sevilla Camino de los Descubrimientos, s/n. Sevilla, 41092, Spain contreras@us.es

Santiago Díaz-Madrigal

Departamento de Matemática Aplicada II Escuela Técnica Superior de Ingenieros, Universidad de Sevilla Camino de los Descubrimientos, s/n. Sevilla, 41092, Spain madrigal@us.es

Pavel Gumenyuk Department of Mathematics University of Bergen Johannes Brunsgate 12 Bergen 5008, Norway Pavel.Gumenyuk@math.uib.no

M. C. and S.D.-M. are partially supported by the Ministerio de Ciencia e Innovación and the European Union (FEDER), project MTM2006-14449-C02-01, by Consejería de Educación y Ciencia de la Junta de Andalucia and by the ESF Networking Programme "Harmonic and Complex Analysis and its Applications". P. G. is partially supported by the ESF Networking Programme "Harmonic and Complex Analysis and its Applications", the Research Council of Norway and the Russian Foundation for Basic Research (grant \#07-01-00120). 